\title{
Performance energética de una vivienda social en Argentina y su rehabilitación basada en simulación térmica
}

\author{
Energy performance of a social dwelling in Argentina and \\ its retrofitting based on thermal simulation
}

\section{Maria Pia Mazzocco \\ Celina Filippín \\ Halimi Sulaiman \\ Silvana Flores Larsen}

\section{Resumen}

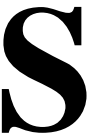

e estudia el comportamiento térmico-energético de una vivienda social en la región central de Argentina. El objetivo es evaluar energéticamente la vivienda para un período histórico entre 1960 y 2011 y obtener el modelo termo físico a través de la simulación con ECOTECT y

SIMEDIF para proponer su rehabilitación. La disponibilidad de datos reales del consumo de energía permitió la calibración a través de la simulación y la obtención del modelo, base de la rehabilitación orientada a la conservación y a la solarización a través de la incorporación de aislación térmica en la envolvente y un aumento del $10,8 \%$ al 20,3\% del área vidriada colectora al norte. Los resultados muestran que la rehabilitación energética permite, además de satisfacer las recomendaciones de las Normas IRAM, mejorar el nivel del etiquetado energético con ambientes térmicamente confortables. A partir de la intervención energética la energía contenida se incrementa un 4,3 y un 7,1\% para alcanzar el Nivel B y A de la Norma IRAM, respectivamente con un ahorro de energía del 53,7 y del 85,6\% en la calefacción $(\mathrm{H})$ de los espacios. El consumo anual por metro cuadrado de superficie útil es de $34,9 \mathrm{kWh} / \mathrm{m}^{2}$, valor auspicioso aún no pudiendo alcanzar el Nivel Passivehouse.

Palablas-clave: Performance energética. Vivienda compacta. Simulación energética. Confort térmico. Rehabilitación energética.

1 Maria Pia Mazzocco

${ }^{1}$ Arquitecta Córdoba - Argentina

${ }^{2}$ Celina Filippín

${ }^{2}$ Consejo Nacional de Investigaciones Científicas y Técnicas Santa Rosa - La Pampa - Argentina

${ }^{3},{ }^{4}$ Halimi Sulaiman ${ }^{3}$ Consejo Nacional de Investigaciones Científicas y Técnicas ${ }^{4}$ Centro Experimental de Vivienda Económica Córdoba - Argentina

${ }^{5}$ Silvana Flores Larsen ${ }^{5}$ Universidad Nacional de Salta Salta - Argentina

Recebido em 15/11/17 Aceito em 29/12/17

\section{Abstract}

This study is concerned with the thermal- energy behaviour of a social dwelling in the central region of Argentina. The aim is to evaluate the energy consumption of the dwelling during the historical period between 1960 and 2011 and to obtain the thermo-physical model through simulation with ECOTECT and SIMEDIF. The availability of real data on energy consumption allowed the calibration through simulation and the design of the model. The retrofitting guidelines aimed at energy conservation and solar collection through the incorporation of thermal insulation in the envelope and an increase from $10.8 \%$ to $20.3 \%$ of the north facing solar glazing area were applied. The results showed that, in addition to meetingthe recommendations of the regulations in force, energy retrofitting leads to the achievement of the objective of this study, improvingthe level of energy labelling with thermally comfortable environments. From an energy intervention perspective, the contained energy increases by 4.3 and $7.1 \%$, reaching Level B and A of the IRAM Standard, respectively, with heating energy savings of 53.7 and $85.6 \%$. The annual consumption per square meter of usable area is $34.9 \mathrm{kWh} / \mathrm{m}^{2}$, which is an interesting value, even though it does not yet reach that of the passive house level.

Keywords: Energy performance. Single house. Energy simulation. Thermal comfort. Energy retrofitting. 


\section{Introducción}

Los edificios son manifestaciones de las innovaciones técnicas y dan cobijo. Las tecnologías empleadas en proyectos y ejecución de esos edificios tienen consecuencias inmediatas en cuanto al consumo de energía (BEHLING; BEHLING, 2002). Santamouris et al. (2006) describen los impactos negativos del sector de la construcción, entre ellos: es el mayor consumidor de energía (alrededor del 30-40\% del consumo mundial) y afecta el ambiente (consume recursos y genera residuos y polución). Zuo y Zhao (2014) argumentan que mientras los edificios satisfacen necesidades humanas y generan innumerables beneficios para la sociedad, también han tenido influencias negativas sobre el medio ambiente durante las últimas décadas. Thormark (2002) sostiene que la construcción emite un $38 \%$ de gases de efecto invernadero, y producir edificios con un impacto ambiental mínimo es un desafío importante para satisfacer las necesidades de una población creciente. Con un aumento previsto de la población de 2.500 millones de personas en 2050 , el uso de la energía en el sector presionará adicionalmente el sistema energético (INTERNATIONAL..., 2013).

En la actualidad hay un interés creciente en el estudio del uso de la energía en los edificios durante su vida útil. El sector residencial tiene un papel fundamental en la demanda energética y el consumo, que fluctúa entre un $20 \%$ y un $35 \%$ para países desarrollados y en desarrollo, respectivamente (NEJAT el al., 2015). Según el Balance Energético Nacional (SECRETARÍA..., 2012), Argentina alcanza un 23\%. Distintos estudios demuestran que la instancia de operación y uso del edificio representa el principal consumo de energía en general (COLE, 1996; ADALBERT, 1997). Sartori y Hestnes (2007) afirman que la energía operativa representa la mayor participación: alrededor del 90 al 95\%, incluso considerando sólo la demanda de energía en calefacción. Filippin, Sipowiczy Flores Larsen (2013) estimaron un valor del 87,5\%. La energía necesaria para la operación del edificio puede decrecer considerablemente si se mejora la resistencia térmica de la envolvente (NIELSEN et al., 1995; WINTER; HESTNES, 1999). Esteves, Gelardi y Mercado (2014) evalúan los costos económicos y los energéticos (energía incorporada) de tecnologías constructivas en Mendoza y consideran que la incorporación de materiales aislantes térmicos en las envolventes de los edificios permite ahorros sustanciales de energía, lo cual mejora las condiciones de confort de los usuarios y la matriz energética actual.
Nejat et al. (2015) afirman que el sector tiene un gran potencial en cuanto al mejoramiento de su eficiencia energética. Ma et al. (2012) sostienen que la remodelación de edificios existentes ofrece oportunidades significativas para reducir el consumo global de energía y las emisiones de gases de efecto invernadero. Esto se está considerando como uno de los principales enfoques para lograr la sostenibilidad en el entorno construido a un costo relativamente bajo y con altas tasas de recuperación de la inversión.

En este contexto, el objetivo principal del presente trabajo es evaluar energéticamente una vivienda social, de tecnología convencional en un clima templado frío de Argentina y, mediante simulación, estudiar la rehabilitación energética de acuerdo a recomendaciones de las Normas IRAM para minimizar el consumo de energía para ambientes térmicamente aceptables.

\section{Metodología}

La Figura 1 muestra un mapa conceptual que detalla el proceso para alcanzar el objetivo general del presente trabajo.

\section{Caracterización climática de la región en estudio}

El lugar de implantación del caso de estudio es la ciudad de Santa Rosa (latitud: 36 $27^{\circ}$ - longitud: $64^{\circ} 27^{\prime}$ - ASNM 182m), capital de la provincia de La Pampa, en la región central de la provincia con clima templado árido y en la zona bioambiental IIIa (INSTITUTO...,1996). En la ciudad, el coeficiente de variabilidad entre los valores promedios decádicos de temperatura, humedad relativa y velocidad de viento oscilan sólo entre 1,7 y 13,6\%. El desvío estándar anual entre décadas es de: $0,4-0,4-0,7^{\circ} \mathrm{C}$ para cada valor de temperatura, 2,6 para la humedad relativa $(\%) \mathrm{y}$ $1,1 \mathrm{~km} / \mathrm{h}$ para velocidad del viento (BELMONTE et al., 2017).

Los veranos presentan temperaturas máximas medias de $31,9{ }^{\circ} \mathrm{C}$ e inviernos de $1,4{ }^{\circ} \mathrm{C}$ con máximas medias de $15,1^{\circ} \mathrm{C}$. La amplitud térmica diaria elevada es una característica importante del clima local, como así también los días claros, donde el aprovechamiento solar es óptimo. Las temperaturas de diseño para invierno y verano se muestran en la Tabla 1.

Respecto a las recomendaciones de diseño, salvo diciembre, enero y febrero el resto de los meses necesita calentamiento solar para lograr confort. Julio requiere además calor auxiliar. 
Figura 1 - Mapa conceptual de la metodología propuesta ${ }^{1}$

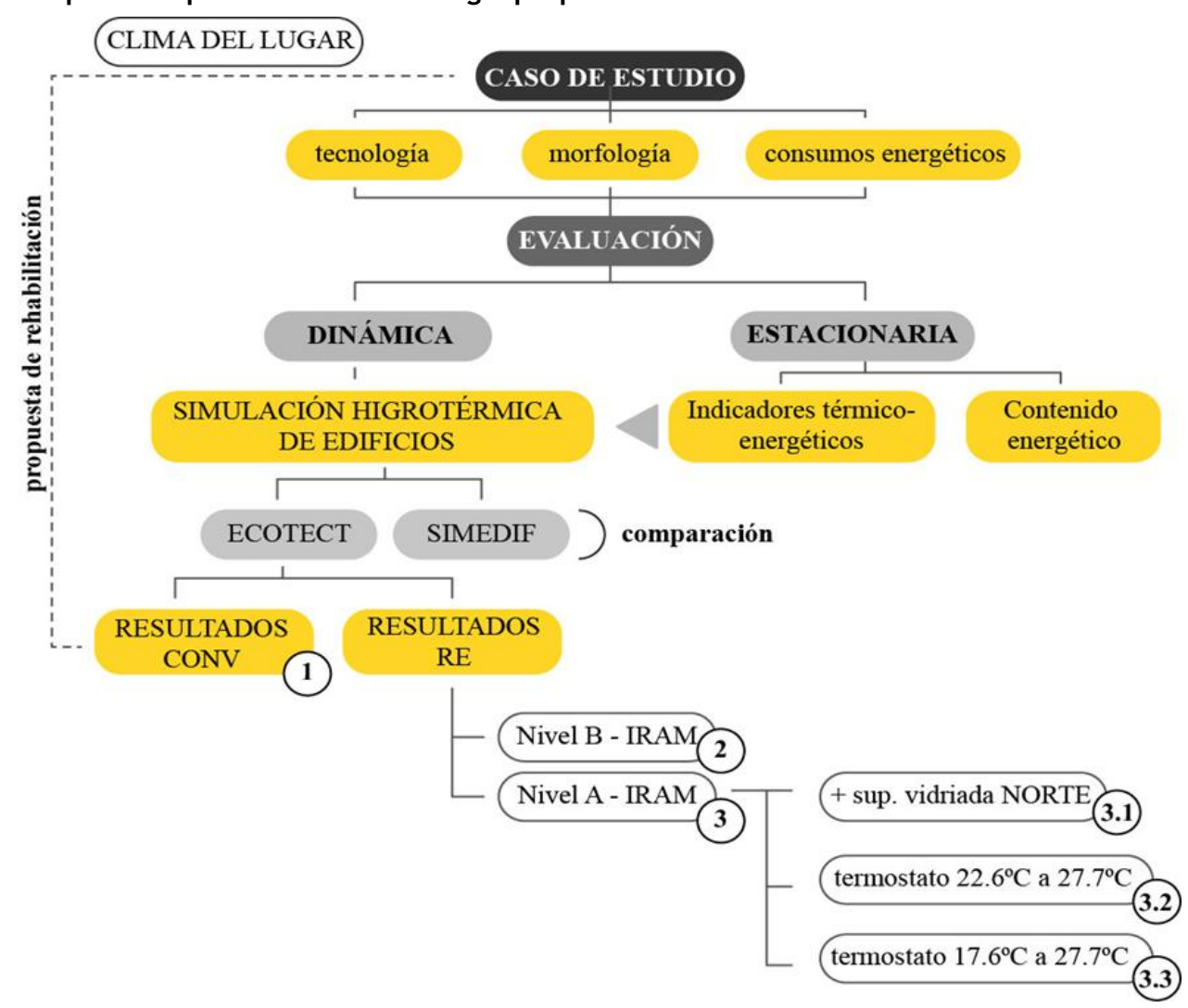

Tabla 1 - Clima de la ciudad de Santa Rosa, provincia de La Pampa

\begin{tabular}{|c|c|c|c|c|}
\hline \multicolumn{2}{|c|}{ Santa Rosa, Latitud: $-36.57^{\circ} \mathrm{S}$, Longitud: $64,27^{\circ}$; ASNM: $189 \mathrm{~m}$} & Anual & Enero & Julio \\
\hline \multirow{5}{*}{ Temperatura $\left({ }^{\circ} \mathrm{C}\right)$} & Máxima media & 23,4 & 31,9 & 15,1 \\
\hline & Mínima media & 8,1 & 15,0 & 1,4 \\
\hline & Media & 15,5 & 23,8 & 7,6 \\
\hline & Mínima absoluta & & & -11.2 \\
\hline & Máxima absoluta & & 41,9 & \\
\hline \multicolumn{2}{|c|}{ Temperatura de diseño mínimo $\left({ }^{\circ} \mathrm{C}\right)$} & & & $-6,0$ \\
\hline \multicolumn{2}{|c|}{ Temperatura de diseño máximo $\left({ }^{\circ} \mathrm{C}\right)$} & & 38,8 & \\
\hline \multicolumn{2}{|c|}{ Velocidadmedia de viento $(\mathrm{km} / \mathrm{h})$} & 12,0 & 14,0 & 11,0 \\
\hline \multicolumn{2}{|c|}{ Radiación global horizontal $\left(\mathrm{MJ} / \mathrm{m}^{2}\right) *$} & 16,30 & 23,98 & 8,09 \\
\hline \multicolumn{2}{|c|}{ Humedad relativa $(\%)$} & 68 & 56 & 78 \\
\hline \multirow{2}{*}{ Grados día de calefacción } & $22,0^{\circ} \mathrm{C}$ & \multirow{2}{*}{\multicolumn{2}{|c|}{$\begin{array}{l}2367,0 \\
1865,0\end{array}$}} & 3230 \\
\hline & $20,0^{\circ} \mathrm{C}$ & & & 323,0 \\
\hline \multicolumn{2}{|c|}{ Grados días de enfriamiento (base $23^{\circ} \mathrm{C}$ ) } & 128,0 & 55,0 & \\
\hline \multicolumn{2}{|c|}{ Precipitaciones $(\mathrm{mm})$} & 631,0 & 69,0 & 15,0 \\
\hline
\end{tabular}

Fuente: Vergara y Casagrande (2012) y *Grossi Gallegos y Riguini (2007).

${ }^{1}$ En adelante la vivienda convencional en estudio se designa CONV, y la rehabilitada RE. 


\section{Descripción tipológica y tecnológica de la vivienda en estudio}

Para llevar adelante el trabajo se toma como caso de estudio una vivienda convencional compacta construida en el año 1960 correspondiente a la tipología "cajón" entre medianeras, típica de la ciudad de Santa Rosa en muy buen estado de conservación (Figura 2). La imagen aérea muestra que la ciudad con 110.000 habitantes es de baja densidad de edificación con predominio de la casa individual. En la Tabla 2 se muestran algunos parámetros dimensionales. Los muros exteriores son de ladrillo común de $0,30 \mathrm{~m}$ revocado en ambas caras y pintados color beige hacia el exterior y blanco hacia el interior, las paredes interiores son de ladrillo común revocado de $0,15 \mathrm{~m}$ de espesor y la envolvente horizontal es una cubierta tradicional compuesta por losa de viguetas pretensadas de hormigón armado, un contrapiso de pendiente de hormigón de cascotes y membrana hidrófuga.

En la Figura 3 se muestra la disposición de los locales y el rango de sombras para el 21 de junio (izquierda) y para el 21 de diciembre (derecha) de 9:00 a 18:00hs para ambos días. El área transparente de ganancia solar directa al norte es de un $15,0 \%$ de la pared y corresponde a un $9 \%$ del área pasiva ${ }^{2}$ y al 4,8\% del área útil de la vivienda.

Figura 2 - Ubicación del caso de estudio

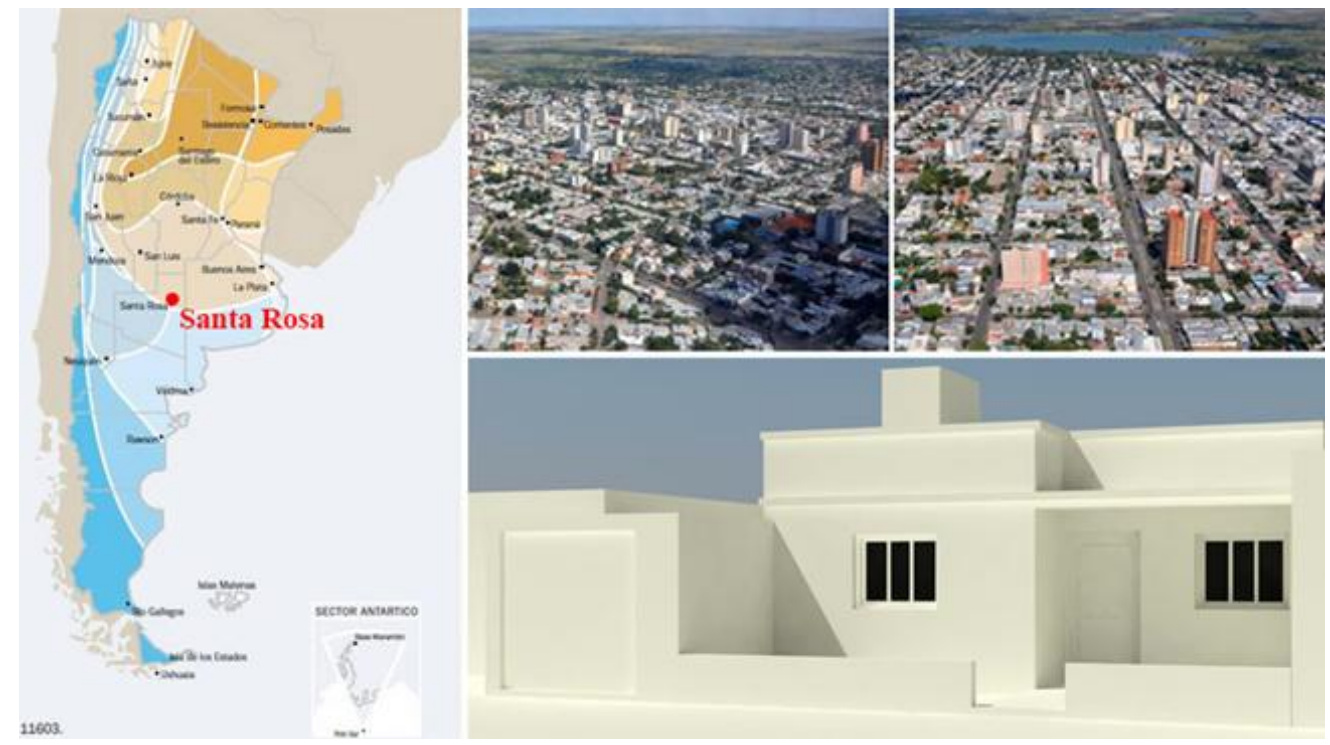

Fuente: Mapa (INSTITUTO..., 1996), fotos aéreas tomadas por el fotógrafo Ramiro Fiorucci, vista 3D (elaboración propia).

Tabla 2 - Datos de la vivienda

\begin{tabular}{|c|c|c|}
\hline \multicolumn{2}{|c|}{ Perímetro (m) } & 32,5 \\
\hline \multicolumn{2}{|c|}{ Área útil $\left(\mathrm{m}^{2}\right)$} & 50,0 \\
\hline \multicolumn{2}{|c|}{ Volumen $\left(\mathrm{m}^{3}\right)$} & 140,0 \\
\hline $\begin{array}{c}\text { Área de } \\
\text { envolvente }\left(\mathrm{m}^{2}\right)\end{array}$ & $\begin{array}{l}\text { pared } \\
\text { Techo } \\
\text { Vano }\end{array}$ & $\begin{array}{c}58,0 \\
50,0 \\
7,2\end{array}$ \\
\hline
\end{tabular}

${ }^{2}$ El método LT define a la zona pasiva como aquella que puede ser iluminada y ventilada naturalmente y puede ser calefaccionada a través de ganancia solar (GOULDING; LEWIS; STEAMERS, 1994).

218 Mazzocco, M. P.; Filippín, C.; Sulaiman, H.; Larsen, S. F. 
Figura 3- Planta y asoleamiento en invierno (izquierda) y verano (derecha)

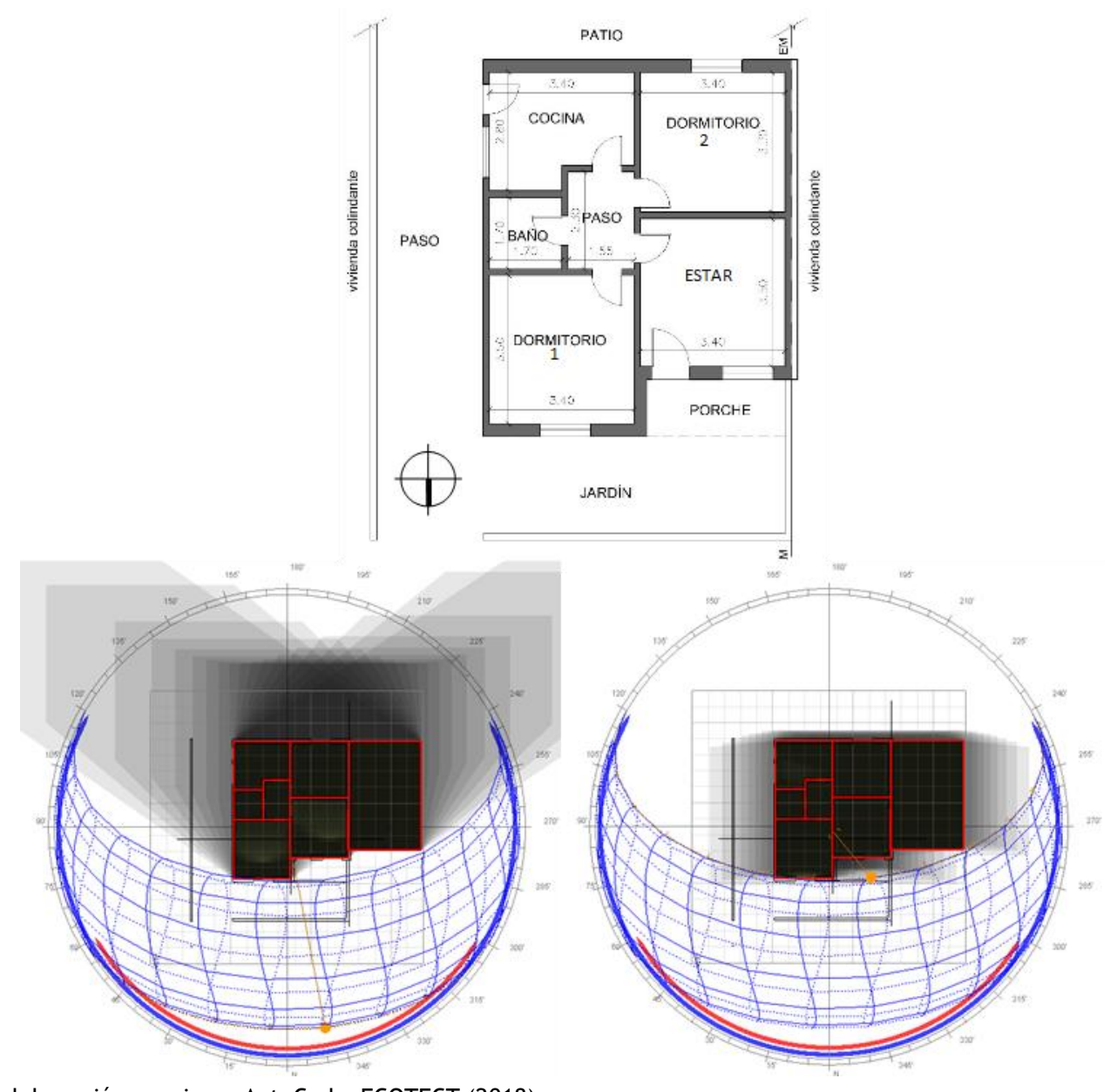

Fuente: elaboración propia en AutoCad y ECOTECT (2018).

\section{Evaluación estacionaria}

La evaluación estacionaria comprende el cálculo de la transmitancia térmica K (INSTITUTO..., 1996), amortiguamiento y retardo. Se emplean planillas pre-configuradas en Excel (GONZALO, 2003) y para establecer los valores de retardo (hs) y amortiguamiento se utiliza el programa Dynamic-Thermal-Properties-Calculator (ver-1-0) herramienta libre $y$ gratuita (CONCRETE CENTRE; ARUP, 2017). Se calcula en función del coeficiente volumétrico $G$ de pérdida de calor $^{3}$ (INSTITUTO..., 2011)), la carga térmica anual de calefacción $Q^{4}$. Se valora el $F A E P^{5}$ (ESTEVES; OLIVA; GELARDI, 1997-1999; ESTEVES;

\footnotetext{
${ }^{3}$ Flujo de calor que pierde un local calefaccionado por unidad de volumen y por unidad de diferencia de temperatura, en régimen estacionario $\left(\mathrm{W} / \mathrm{m}^{3 \circ} \mathrm{C}\right)$.

${ }^{4}$ Energía que debe suplir el equipo de calefacción para mantener constante la temperatura interior durante un período dado, sin considerar las ganancias $(\mathrm{kWh})$.

${ }^{5} \mathrm{FAEP}=$ Superficie $_{\text {techo }}+$ Superficie $_{\text {muro }}+$ Superficie $_{\text {ventana }}+$ Superficie puerta/superficie cubierta a calefaccionar
}

GELARDI, 2010) indicador que relaciona el área de la envolvente con la superficie útil de la vivienda. Con éste, el diseñador puede actuar para optimizar el comportamiento térmico del edificio considerando la construcción y su valor monetario. Cuanto más próximo a 2 sea su valor, menor será la superficie de la envolvente, menores serán los costos de construcción y la superficie de intercambio de la vivienda con el exterior. Los autores consideran que, con ese valor, la forma es energéticamente eficiente. Mascaró (1983) sugiere el índice: $I_{c}{ }^{6}$ que representa el grado de compacidad de la planta del edificio como un indicador morfológico adecuado desde el punto de vista económico. A mayor compacidad, menor costo. Para estimar la energía contenida ${ }^{7}$ se toma como referencia la clasificación por rubro según la

\footnotetext{
${ }^{6} \mathrm{I}_{C}=$ relación entre el perímetro del círculo y el perímetro del proyecto.

${ }^{7}$ Energíacontenida: suma de toda la energía necesaria para fabricar un bien. Puede no incluir la energía de la materia prima
} (SARTORI; HESTNES, 2007). 
composición del costo total de la vivienda que define Mascaró (1983). Se consideran los componentes tecnológicos (elevación y cimientos), terminaciones y carpintería que integran los planos horizontales y verticales que corresponden a un $71,2 \%$ del costo total de la construcción.

Respecto al consumo de energía, el análisis se centra sobre todo en la fase de funcionamiento del edificio (energía operativa ${ }^{8}$ ) y la fase de energía contenida. Las fases de renovación y demolición no se tienen en cuenta ya que no hubo remoción de materiales y la vivienda presenta muy buen estado de conservación. Tampoco se considera la pérdida de material en el proceso de construcción y las redes de infraestructura (instalaciones eléctricas, sanitarias, y de gas). Para estimar el total de energía consumida por la vivienda se considera el año 1 período de construcción y del 2 al 50 el período de operación del edificio de acuerdo con Zabalza Bribianet al. (2009) y Crawford (2012) quienes plantean una expectativa de vida aproximada a los 50/75 años. Luego, se torna necesaria una intervención general. Esta resultará necesaria en relación del edificio que se trate y su función, su uso y también el mantenimiento que se haya realizado a lo largo de su vida (GONZALEZ MORA, 2005). Zabalza Bribián et al. (2009) argumentan en su estudio, y en un método simplificado de análisis de ciclo de vida, que la vida útil de una casa es de 50 años para todos los materiales, sistemas y componentes. En el presente trabajo se considera un valor central según Crawford (2012) y Zabalza Bribian et al. (2009), coincidente con 1961 (año 1) y 2011 (últimos datos de energía consumida disponibles).

\section{Evaluación dinámica}

Clarke (2001) considera que la simulación representa una posible solución al dilema de la complejidad edilicia al permitir evaluaciones integrales e integradas de las opciones de diseño, admite la emulación de realidades futuras, la capacidad de apreciar el comportamiento poco fiable de un sistema y, por lo tanto, tomar medidas sutiles para mejorar el rendimiento a través de criterios relevantes. Concluye que la simulación representa un cambio de paradigma de gran potencial en el diseño, con resultados que mejor se ajusten a las aspiraciones de prácticas sostenibles, la protección del medio ambiente y la mitigación del cambio climático. El autor argumenta también que es una herramienta

${ }^{8}$ Energía operativa: energía utilizada en la construcción durante su fase operativa, en cuanto a: calefacción, refrigeración, electrodomésticos (SARTORI; HESTNES, 2007). indispensable en el análisis de la performance del edificio en su etapa post construcción. En el presente trabajo, para llevar adelante la simulación energética, se modela la vivienda con ECOTECT ${ }^{9}$ y Simedif para Windows ${ }^{10}$. ECOTECT permite un análisis integral y en simultáneo de situaciones de asoleamiento, configuración geométrica y tecnológica sobre un modelo en tres dimensiones que se trabaja dentro del mismo software, en el cual los cambios y el desarrollo de alternativas pueden visualizarse de manera eficaz. Distintos autores han empleado ECOTECT para calibrar datos medidos $\mathrm{y}$ simulados en la región. Boutet, Hernández y Jacobo (2012) y Di Bernardo, Filippín y Pipa (2011) concluyen en su investigación que es razonable el ajuste obtenido entre datos medidos y simulados. Harish y Kumar (2016) describen en su trabajo la capacidad de diferentes programas de simulación, entre ellos ECOTECT. Ellos consideran que hay características que están implementadas, como: la descripción geométrica, la consideración de las ganancias internas y solares, la sombra y el tipo de cielo y las propiedades de los elementos constructivos. Se decide también contrastar resultados con SIMEDIF, software desarrollado en el INENCO (Instituto de Investigación de Energía No Convencional, Argentina) para el diseño de edificios pasivos y simulación de comportamiento térmico de edificios en régimen transitorio (FLORES LARSEN; LESINO, 2001). El código ha sido ampliamente validado a lo largo de años de trabajo experimental en Argentina (FLORES LARSEN et al., 2008; FLORES LARSEN; FILIPPÍN; LESINO, 2009; FILIPPÍN; FLORES LARSEN; LOPEZ, 2008). Con ECOTECT se evalúa el comportamiento térmico y se estima el consumo de energía anual, y para días críticos de invierno y verano. Con SIMEDIF se estima el consumo diario de energía para comparar con valores reales medidos y con los valores resultantes en ECOTECT. Se considera para las condiciones internas de diseño un clo de 1 (ropa

\footnotetext{
${ }^{9}$ ECOTECT utiliza un método de cálculo simplificado basado en el Método de Admitancias del Chartered Institute of Building Services Engineers (CIBSE Admittance Method), para determinar temperaturas internas y cargas térmicas, aplicando unalgoritmo térmico flexible, sin restricciones en cuanto a la geometría del edificio o el número de zonas térmicas. Aplica la admitancia de los elementos constructivos y los factores de retraso térmico y atenuación térmica de materiales para definir la respuesta en régimen transitorio (AUTODESK, 2011).

${ }^{10}$ SIMEDIF para Windows permite realizar la simulación térmica de edificios en régimen estacionario y dinámico(FLORES LARSEN; LESINO, 2001). Evalúa el comportamiento de edificios nuevos o construidos frente a variaciones climáticas, orientación, materialidad, permitiendo detectar la influencia de las mejoras y el confort interior.
} 
liviana) y una humedad del $60 \%$ con una velocidad de viento $0,5 \mathrm{~m} / \mathrm{seg}$ en todos los locales. Se determina la cantidad de personas por habitación, así como el tipo de actividad y horarios de uso. Para las ganancias internas se tiene en cuenta el aporte de artefactos eléctricos y de iluminación. Para las infiltraciones se considera una tasa de cambio de aire (renovaciones) de 3 (CZAJKOWSKI; GOMEZ, 1994), correspondiente al tipo de abertura existente: de madera poco estanca y una sensibilidad al viento de 0,25 (intercambio de aire/hs) que corresponde a un tipo de abertura razonablemente protegida. El sistema de acondicionamiento adoptado para la simulación es de "modo mixto": sistema de refrigeración y calefacción (HVAC) + ventilación natural cuando las condiciones externas lo permiten según banda de confort establecida entre $24,5^{\circ} \mathrm{C}$ a $27,5^{\circ} \mathrm{C}$ con una eficiencia del sistema del $65 \%$. En un trabajo anterior los resultados del comportamiento térmico energético en invierno de viviendas tecnológica y morfológicamente similares muestran temperaturas promedios interiores medidas entre 16,0 y $21,8^{\circ} \mathrm{C}$ con sus consumos de gas natural promedios diarios entre $0.10 \mathrm{y}$ $0.22 \mathrm{~m}^{3} / \mathrm{m}^{2}$. Endías rigurosos de invierno, la temperatura interior alcanza valores de hasta 25 ${ }^{\circ} \mathrm{C}$ con un consumo diario de gas natural que trepa a los $0.26 \mathrm{~m}^{3} / \mathrm{m}^{2}$. (FILIPPIN; SIPOWICZ; FLORES LARSEN, 2013).

En el presente trabajo, los valores de los consumos de energía medidos permiten contar con una referencia confiable para acordar los valores definidos en la carga de datos e información y así definir el modelo termo-físico de la vivienda con el fin de estudiar el potencial de intervención y la posible rehabilitación energética. El consumo de gas natural destinado a la calefacción de la vivienda en estudio es de $627,5 \mathrm{~m}^{3}$. El consumo diario y el consumo diario $/ \mathrm{m}^{2}$ es de $10,5 \mathrm{~m}^{3} \mathrm{y}$ $0,21 \mathrm{~m}^{3} / \mathrm{m}^{2}$, respectivamente, valor similar al registrado en viviendas monitoreadas. Las Tablas 3 y 4 muestran el consumo promedio mensual y anual de gas natural y energía eléctrica según las facturas correspondientes. El coeficiente de variación entre los años del período considerado (desvío estándar/promedio*100) oscila entre un $10,00 \%$ y un $5,70 \%$ para el consumo de gas natural y de electricidad, respectivamente.

\section{Análisis de resultados Indicadores energéticos resultantes a través de la evaluación estacionaria}

Los cálculos estacionarios corresponden a diferentes Normas IRAM (11601 (INSTITUTO..., 2002)), 11604 (INSTITUTO..., 2011) у 11605 (INSTITUTO..., 1996b)) y de acuerdo a los programas de cálculos descriptos en metodología y cuyos resultados se describen:

(a) envolvente vertical exterior: $\mathrm{K}=2,04 \mathrm{~W} / \mathrm{m}^{2} \mathrm{~K}$, valor que no alcanza el nivel menos exigente de la norma IRAM 11605 (INSTITUTO..., 1996b) (Nivel C para verano, $\mathrm{K}=2 \mathrm{~W} / \mathrm{m}^{2} \mathrm{~K}$ ). El retardo y el amortiguamiento son de $6 \mathrm{hs}$ y 0,18 , respectivamente;

(b) paredes interiores: $\mathrm{K}=2,97 \mathrm{~W} / \mathrm{m}^{2} \mathrm{~K}$;

(c) envolvente horizontal de techo: $\mathrm{K}$ :

$2,03 \mathrm{~W} / \mathrm{m}^{2} \mathrm{~K}$, valor superior a los recomendados por la misma norma (K entre $0,26 \mathrm{~W} / \mathrm{m}^{2} \mathrm{~K}$ y $1 \mathrm{~W} / \mathrm{m}^{2} \mathrm{~K}$, niveles A y $\mathrm{C}$, respectivamente). $\mathrm{El}$ retardo y el amortiguamiento es de 8 hs y 0,16 , respectivamente; $\mathrm{y}$

(d) ventanas: vidrio simple con carpintería de madera con persiana de madera maciza con un $\mathrm{K}$ de 2,79 y $5,10 \mathrm{~W} / \mathrm{m}^{2} \mathrm{~K}$ cuando está cerrada y abierta, respectivamente.

Tabla 3 - Promedio del consumo histórico de gas natural $\left(\mathrm{m}^{3}\right)$ entre 1996 y 2011

\begin{tabular}{|c|c|c|c|c|c|c|c|}
\hline \multicolumn{6}{|c|}{ Bimestres (promedios históricos) } & \multirow{2}{*}{\multicolumn{2}{|c|}{ Anual }} \\
\hline 1 & 2 & 3 & 4 & 5 & 6 & & \\
\hline 68,3 & 115,3 & 591,3 & $\begin{array}{l}836,7(75 \% \\
\text { destinado a } \\
\text { calefacción) }\end{array}$ & 488,2 & 118,5 & $\begin{array}{c}2218,6(67 \% \text { destinado a } \\
\text { calefacción }=1486 \mathrm{~m}^{3}= \\
12482400 \mathrm{kcal}=144801 \mathrm{Wh})\end{array}$ & $\begin{array}{c}\text { Total anual } \\
\text { promedio entre } \\
1996 \text { y } 2011\end{array}$ \\
\hline 15,6 & 31,1 & 138,2 & 108,5 & 138,5 & 20,5 & 229,8 & Desvíoestándar \\
\hline 22,8 & 27,0 & 23,4 & 13,0 & 28,4 & 17,3 & 10,1 & $\begin{array}{l}\text { Coeficiente de } \\
\text { variación (\%) }\end{array}$ \\
\hline
\end{tabular}


Tabla 4 - Promedio del consumo histórico de electricidad (kWh) entre 2000 y 2011

\begin{tabular}{c|c|c|c|c|c|c|c|c|c|c|c|c|c}
\hline $\mathbf{E}$ & $\mathbf{F}$ & $\mathbf{M}$ & $\mathbf{A}$ & $\mathbf{M}$ & $\mathbf{J}$ & $\mathbf{J}$ & $\mathbf{A}$ & $\mathbf{S}$ & $\mathbf{O}$ & $\mathbf{N}$ & $\mathbf{D}$ & \multicolumn{2}{|c}{ Anual } \\
\hline 109,5 & 101,1 & 82,9 & 84,6 & 78,8 & 83,3 & 84,2 & 81,3 & 78,9 & 79,7 & 80,1 & 89,8 & 1064,0 & Promedio \\
\hline 16,4 & 24,1 & 12,2 & 8,7 & 6,7 & 6,4 & 7,2 & 6,0 & 5,4 & 9,2 & 7,4 & 11,7 & 59,0 & $\begin{array}{l}\text { Desvío } \\
\text { estándar }\end{array}$ \\
\hline 15,0 & 23,8 & 14,7 & 10,3 & 8,5 & 7,7 & 8,6 & 7,4 & 6,8 & 11,6 & 9,2 & 13,0 & 5,7 & $\begin{array}{l}\text { Coeficiente } \\
\text { de } \\
\text { variación } \\
(\%)\end{array}$ \\
\hline
\end{tabular}

Tabla 5 - Indicadores energéticos

\begin{tabular}{|c|c|c|c|c|}
\hline $\mathrm{G}\left(\mathrm{W} / \mathrm{m}^{3 \mathrm{o}} \mathrm{C}\right)$ & & & & 3,0 \\
\hline \multirow{6}{*}{$\mathrm{Q}_{\text {auxiliar }}$} & \multirow{3}{*}{$\begin{array}{l}\text { Estimado de acuerdo a } \\
\mathrm{G}\end{array}$} & $\mathrm{kWh} / \mathrm{año} / \mathrm{m}^{2}$ & & 290,0 \\
\hline & & Kerosene (lts) & & 1620,0 \\
\hline & & Gas Natural $(\mathrm{GN}) \mathrm{m}^{3}$ & & 1485,0 \\
\hline & \multirow{3}{*}{$\begin{array}{l}\text { GN medido promedio } \\
\text { durante el período }\end{array}$} & $\mathrm{m}^{3}$ & & 2218,0 \\
\hline & & \multirow{2}{*}{$\begin{array}{l}\text { (67\% destinado a } \\
\text { calefacción) }\end{array}$} & $\mathrm{m}^{3}$ & 1486,0 \\
\hline & & & $\mathrm{kWh} / \mathrm{m}^{2}$ & 289,6 \\
\hline Ic $(\%)$ & & & & 77,0 \\
\hline FAEP & & & & 1,3 \\
\hline
\end{tabular}

De acuerdo a la permeabilidad térmica de la envolvente, el volumen de la vivienda y las renovaciones de aire, el Coeficiente volumétrico $G$ de pérdidas de calor es de $3,0 \mathrm{~W} / \mathrm{m}^{3} \mathrm{~K}$, expresado en Tabla 5, es superior al valor admisible según la Norma IRAM 11601 (INSTITUTO..., 2002) de $1,878 \mathrm{~W} / \mathrm{m}^{3} \mathrm{~K}$. El factor de forma, FAEP, resulta em 1,3, valor inferior al sugerido por Esteves y Gelardi (2010) para edificios energéticamente eficientes, y el índice de compacidad, Ic en 77\% (MASCARÓ, 1983). Ambos indicadores morfológicos son adecuados, no así el Coeficiente volumétrico de pérdidas de calor que amerita un mejoramiento de la resistencia térmica de la envolvente para alcanzar el valor admisible según la Norma.

La Tabla 6 muestra el contenido energético de los rubros más significativos de la vivienda con un total de $8779,50 \mathrm{MJ} / \mathrm{m}^{2}$. En la estimación de la energía contenida no se considera el rubro de infraestructura (instalación eléctrica, sanitaria y de gas). En la Figura 4 se observa la energía contenida en $\mathrm{GJ} / \mathrm{m}^{2}$ de la vivienda en estudioy los valores obtenidos por diferentes autores con un promedio de $5,60 \mathrm{GJ} / \mathrm{m}^{2}$ y un coeficiente de variación (relación entre el promedio y el desvío estándar de la muestra) del 28,60\%.

Con el fin de estimar la participación relativa en el consumo total de energía en el período de vida transcurrido (50 años) es pertinente conocer el valor de la operativa que fue estimada en Filippin et al. (2017) con la denominación de $\mathrm{VA}_{3}$. En la investigación se realizó un análisis retrospectivo del consumo de energía que incluye una muestra de viviendas de tipología compacta y describe la energía operativa para un período de vida transcurrido de 50 años.

En el presente trabajo se sintetizan algunos resultados que permiten estimar la participación relativa de la energía contenida y operativa. Se realiza un análisis de regresión simple a partir de los datos reales bimestrales del consumo del gas natural entre 1996 y $2011\left(\mathrm{~m}^{3}\right)$ (Tabla 1) y del consumo mensual de energía eléctrica $(\mathrm{kWh})$, período 2000-2011 (Tabla 2), sin interrupción en ambos casos. El análisis entre consumo de gas natural (y) y año (x) arroja el modelo que se detalla en Tabla 7. Dado que el p-valor en la tabla ANOVA es inferior a 0,01 , existe una relación estadísticamente significativa entre el consumo de gas natural y años para un nivel de confianza del 99,0\%. El estadístico R-cuadrado indica que el modelo explica un $53,1 \%$ de la variabilidad en el consumo después de la transformación a escala logarítmica para linealizar el modelo. El coeficiente de correlación es igual a $-0,73$ (r según Tabla 7), indicando una relación moderadamente fuerte entre las variables. El modelo ajustado para el consumo de electricidad se observa en la misma tabla. Dado que el p-valor en la tabla ANOVA es inferior a 0,05 , existe relación estadísticamente significativa entre consumo de electricidad y años para un nivel de confianza del 95\%. El estadístico R-cuadrado indica que el modelo explica un $50,96 \%$ de la variabilidad en el consumo. El coeficiente de correlación ( $r$ de la Tabla 6 ) es igual a 0,71 indicando una relación moderadamente fuerte entre las variables. 
Tabla 6 - Estimación de la energía contenida

\begin{tabular}{|c|c|c|}
\hline Componente tecnológico & Energía primaria $\mathrm{MJ} / \mathrm{kg}$ & MJ \\
\hline $\begin{array}{l}\text { Muro de cimiento y elevación de } \\
\text { ladrillomacizo }\end{array}$ & $\begin{array}{l}\text { 2,85 (CEPEDA GUTIÉRREZ; MARDARAS } \\
\text { LARRAÑAGA, 2013) }\end{array}$ & 349404,0 \\
\hline $\begin{array}{l}\text { Encadenado inferior y superior de } \\
\text { hormigón armado }\left(\mathrm{k}=1.65 \mathrm{~W} / \mathrm{m}^{2 \mathrm{o}} \mathrm{C}\right)\end{array}$ & 0,99 & 3564,0 \\
\hline Contrapiso (no se considera solado) & $\begin{array}{l}2,03 \mathrm{MJ} / \mathrm{kg} \\
\text { Valor elaborado por autores de acuerdo a una } \\
\text { dosificación de un HHRP (hormigón } \\
\text { hidráulico reforzado pobre: } 1 \text { cal, } 1 / 8 \text { cemento, } \\
4 \text { arena, } 8 \text { cascotes) }\end{array}$ & 16240,0 \\
\hline $\begin{array}{l}\text { Techo: Cubierta horizontal con } \\
\text { membrana de aluminio sobre estructura } \\
\text { resistente prefabricada }\end{array}$ & $\begin{array}{l}\text { 490,0 MJ/m² }(\text { ESTEVES; GELARDI; } \\
\text { MERCADO, 2014) } \\
\text { + contrapiso: } 325,0 \mathrm{MJ} / \mathrm{m}^{2}\end{array}$ & 48888,0 \\
\hline $\begin{array}{l}\text { Revoque exterior e interior y cielorraso } \\
\left(40 \mathrm{~kg} / \mathrm{m}^{2}\right)\end{array}$ & 1,34 & 18974,0 \\
\hline Pintura & $1,40 \mathrm{MJ} / \mathrm{m}^{2}$ & 496,0 \\
\hline Carpintería & $\begin{array}{l}\text { Marco de madera: } 294,0 \mathrm{MJ} / \mathrm{m}^{2} \text { (ESTEVES; } \\
\text { GELARDI; MERCADO, 2014) }\end{array}$ & 1411,0 \\
\hline \multirow{2}{*}{ TOTAL } & MJ & $\mathrm{MJ} / \mathrm{m}^{2}$ \\
\hline & 438977,0 & 8779,5 \\
\hline
\end{tabular}

Figura 4 - Diferentes valores de energía cotenida $\left(\mathrm{GJ} / \mathrm{m}^{2}\right)$ en viviendas

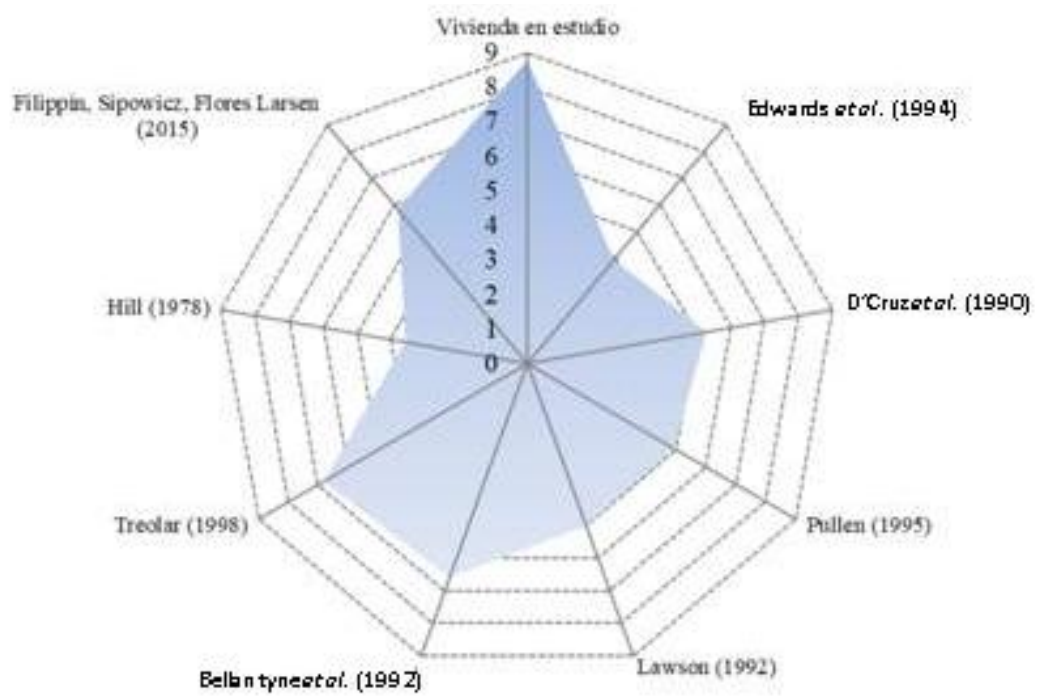

Fuente: gráfico realizado tomando como referencia a Ding (2004) ${ }^{11}$ citado en Dixit et al.(2010). Dingen sus Tesis Doctoral en la Tabla 3.1 resume los estudios de distintos autores de energía contendida $/ \mathrm{m}^{2}$ en el sector residencial excepto Filippín, Sipowicz y Flores Larsen (2013) y Vivienda en estudio.

Tabla 7 - Modelo de regresión lineal simple: consumo de energía vs. Año. r: coeficiente de correlación

\begin{tabular}{|c|c|c|c|c|c|c|c|}
\hline \multicolumn{4}{|c|}{$\begin{array}{l}\text { Consumo de gas natural (Período: 1996- } \\
\text { 2011) }\end{array}$} & \multicolumn{4}{|c|}{$\begin{array}{l}\text { Consumo de energía eléctrica (Período: 2000- } \\
\text { 2011) }\end{array}$} \\
\hline Modelo & $r$ & \begin{tabular}{l|l}
$R^{2}$ & \\
\end{tabular} & $P$-valor & Modelo & $r$ & $R^{2}$ & $P$-valor \\
\hline $\begin{array}{l}\exp (57.2164- \\
99220.0 / x)\end{array}$ & $\overline{-}$ & 53,10 & 0,0014 & $\begin{array}{l}-26789,30+ \\
13,8667 * x\end{array}$ & $\begin{array}{c}0,7 \\
1\end{array}$ & 51 & 0,0241 \\
\hline
\end{tabular}

${ }^{11}$ DING, G. K.The Development of a Multi-Criteria Approach For the Measurement of Sustainable Performance For Built Projects and Facilities. Sydney, 2004. Thesis (Doctor of Philosophy) - University of Technology, Sydney, 2004. 
Con el fin de estimar la participación relativa en el consumo total de energía en el período de vida transcurrido (50 años) es pertinente conocer el valor de la operativa que fue estimada en Filippin et al. (2017) con la denominación de VA3. En la investigación se realizó un análisis retrospectivo del consumo de energía que incluye una muestra de viviendas de tipología compacta y describe la energía operativa para un período de vida transcurrido de 50 años.

En el presente trabajo se sintetizan algunos resultados que permiten estimar la participación relativa de la energía contenida y operativa. Se realiza un análisis de regresión simple a partir de los datos reales bimestrales del consumo del gas natural entre 1996 y 2011 (m3) (Tabla 1) y del consumo mensual de energía eléctrica $(\mathrm{kWh})$, período 2000-2011 (Tabla 2), sin interrupción en ambos casos. El análisis entre consumo de gas natural (y) y año (x) arroja el modelo que se detalla en Tabla 7. Dado que el p-valor en la tabla ANOVA es inferior a 0,01 , existe una relación estadísticamente significativa entre el consumo de gas natural y años para un nivel de confianza del 99,0\%. El estadístico R-cuadrado indica que el modelo explica un $53,1 \%$ de la variabilidad en el consumo después de la transformación a escala logarítmica para linealizar el modelo. El coeficiente de correlación es igual a $-0,73$ ( $r$ según Tabla 7), indicando una relación moderadamente fuerte entre las variables. El modelo ajustado para el consumo de electricidad se observa en la misma tabla. Dado que el p-valor en la tabla ANOVA es inferior a 0,05 , existe relación estadísticamente significativa entre consumo de electricidad y años para un nivel de confianza del $95 \%$. El estadístico R-cuadrado indica que el modelo explica un $50,96 \%$ de la variabilidad en el consumo. El coeficiente de correlación ( $\mathrm{r}$ de la Tabla 6 ) es igual a 0,71 indicando una relación moderadamente fuerte entre las variables.

La energía operativa muestra un valor acumulado $\mathrm{y}$ un valor promedio de $2.855 .890,6 \mathrm{MJ} / \mathrm{m}^{2}$ y $57.117,8 \mathrm{MJ} / \mathrm{m}^{2}$ respectivamente e incluye el total de energía para satisfacer los requerimientos de calefacción $(58,2 \%)$, cocción de alimentos y calentamiento de agua $(36,8 \%)$ e iluminación y equipamiento (5,0\%) (FILIPPIN et al., 2017). De acuerdo a ambos resultados, la energía contenida absorbe un $1,96 \%$ de la energía total en el período transcurrido.

\section{Performance energética a través de la simulación térmica}

En un primer paso se simula el comportamiento de la vivienda convencional (CONV) con ECOTECT para estimar la energía necesaria para mantener los niveles de confort durante todo el año. El modelo para simulación incluye la vivienda colindante ya que en este caso evita la radiación solar directa y difusa sobre la fachada Oeste. En la Tabla 8 se detallan los parámetros considerados en la simulación térmica en ambos programas. En cuanto a las renovaciones, se estiman según largo de juntas, caudal de infiltración para una carpintería normal (INSTITUTO..., 2001), coeficiente de corrección según características del entorno, velocidad del viento y volumen del local. Para el estar y ambos dormitorios el valor es de $2,8 \mathrm{~m}^{3} / \mathrm{h}$ el cual se adopta para baño y cocina (YAÑEZ PARAREDA, 1982). El CLO se considera segúnNorma ISO 7730 (INTERNATIONAL..., 2005). La velocidad de viento se estima según Czajkowski y Gomez (1994). Los mismos autores estiman que velocidades entre 0,25 a $0,5 \mathrm{~m} / \mathrm{s}$ generan un ambiente agradable. En cuanto a los componentes tecnológicos, se introdujeron los valores previamente calculados. La Tabla 9 detalla los valores de las variables climáticas usadas según Vergara y Casagrande (2012) y Grossi Gallegos y Righini (2007).

El consumo total anual de energía destinado a calefacción $(\mathrm{H})$ para una banda de confort entre $24,5{ }^{\circ} \mathrm{C}$ a $27,5^{\circ} \mathrm{C}$ es de $14.474,1 \mathrm{kWh}$ cuya conversión acusa $1.482,0 \mathrm{~m}^{3}$ de gas natural, (promedio histórico auditado: $1486 \mathrm{~m}^{3}$ según Tabla 2). El 97,0\% y el 3,0\% del consumo de energía total anual $(14.934,0 \mathrm{kWh})$ para $\mathrm{H}$ y refrigeración (AC) de la vivienda respectivamente. El consumo de energía $/ \mathrm{m}^{2}$ es de $289,6 \mathrm{kWh} / \mathrm{m}^{2}$ para $\mathrm{H}$ y $9,2 \mathrm{kWh} / \mathrm{m}^{2}$ para AC. Respecto al consumo de energía en el bimestre más frío (julio-agosto) el valor obtenido en la simulación es de $687,0 \mathrm{~m}^{3}$, un $9,4 \%$ superior al valor real auditado $\left(627,5 \mathrm{~m}^{3}\right)$. La Figura 5 muestra la estacionalidad del consumo de energía para mantener los niveles de confort establecidos anteriormente.

En la Tabla 10 se observan las pérdidas y ganancias en $\mathrm{kWh}$ a través del sistema HVAC, la envolvente, las superficies vidriadas (ganancia solar directa), ventilaciones e infiltraciones, usuarios y artefactos (ganancias internas). 
Tabla 8 - Parámetros considerados para la simulación

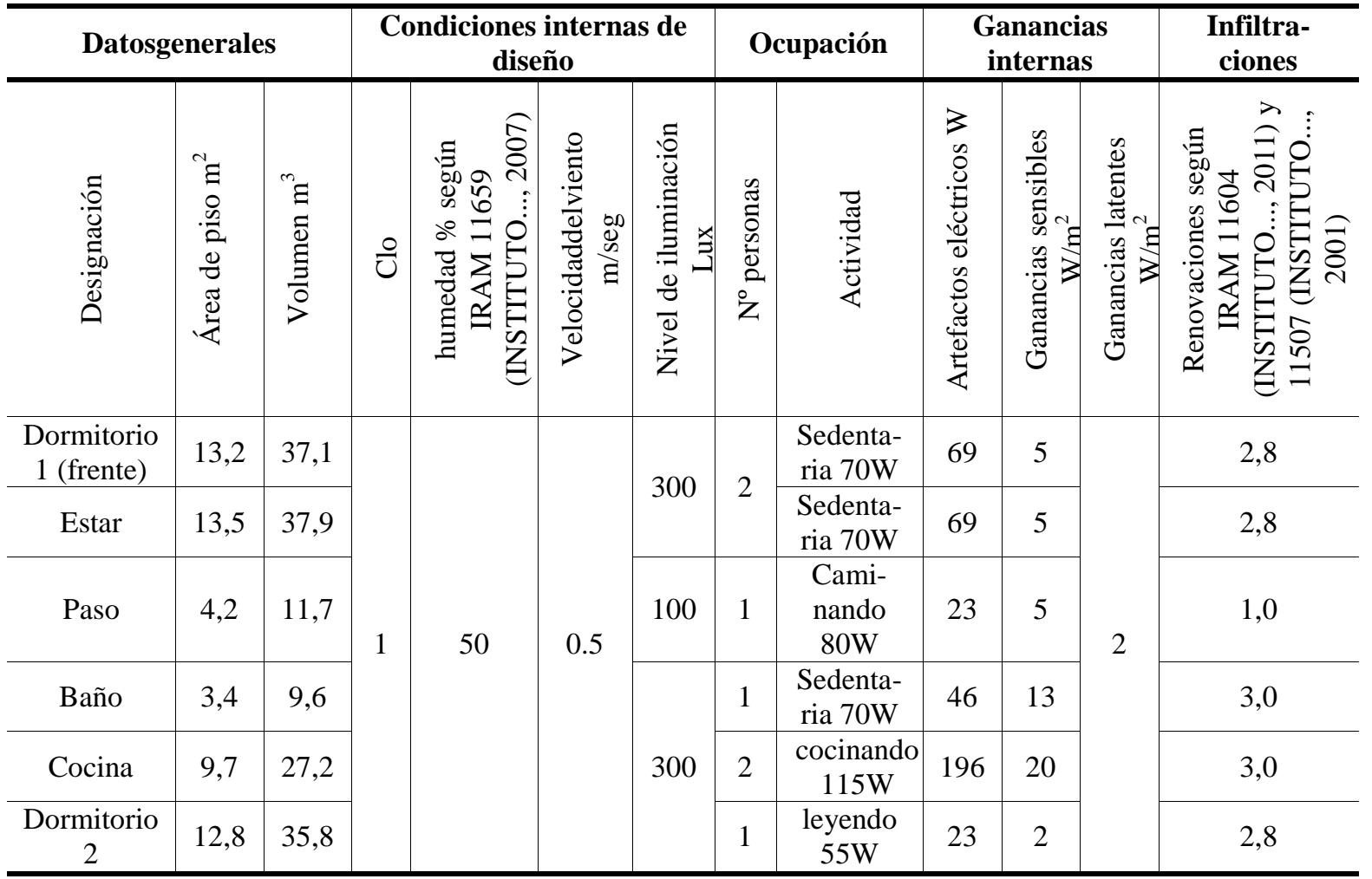

Tabla 9 - Variables climáticas promedio entre 1960 y 2011

\begin{tabular}{|c|c|c|c|c|c|c|c|c|c|c|c|c|c|c|}
\hline \multicolumn{2}{|c|}{ Variables } & $\mathbf{E}$ & $\mathbf{F}$ & $\mathbf{M}$ & $\mathbf{A}$ & $\mathbf{M}$ & $\mathbf{J}$ & $\mathbf{J}$ & $\mathbf{A}$ & $\mathbf{S}$ & $\mathbf{O}$ & $\mathbf{N}$ & D & Anual \\
\hline \multirow{3}{*}{ 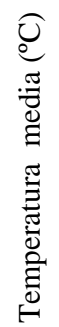 } & máxima & 31,8 & 29,9 & 26,6 & 22,0 & 17,4 & 14,1 & 14,0 & 16,6 & 19,8 & 23,6 & 27,1 & 30,3 & 22,8 \\
\hline & media & 23,8 & 22,1 & 19,5 & 15,1 & 11,2 & 8,4 & 7,8 & 9,6 & 12,4 & 16,4 & 19,5 & 22,5 & 15,7 \\
\hline & mínima & 15,7 & 14,3 & 12,5 & 8,3 & 4,9 & 2,7 & 1,5 & 2,7 & 5,1 & 9,2 & 11,8 & 14,7 & 8,6 \\
\hline \multicolumn{2}{|c|}{ HR (\%) } & 65,0 & 66,0 & 71,0 & 76,0 & 79,0 & 79,0 & 77,0 & 69,0 & 68,0 & 68,0 & 64,0 & 62,0 & 70,0 \\
\hline \multicolumn{2}{|c|}{$\begin{array}{l}\text { Irradiación } \\
\text { solar global } \\
\text { diaria } \\
\text { promedio } \\
\left(\mathrm{Wh} / \mathrm{m}^{2}\right)\end{array}$} & 6550 & 6000 & 4500 & 3000 & 2500 & 1500 & 2000 & 3000 & 4000 & 5000 & 6000 & 7000 & 4254 \\
\hline
\end{tabular}


Figura 5 - Carga térmica de H y AC mensual y anual en kWh (eje y

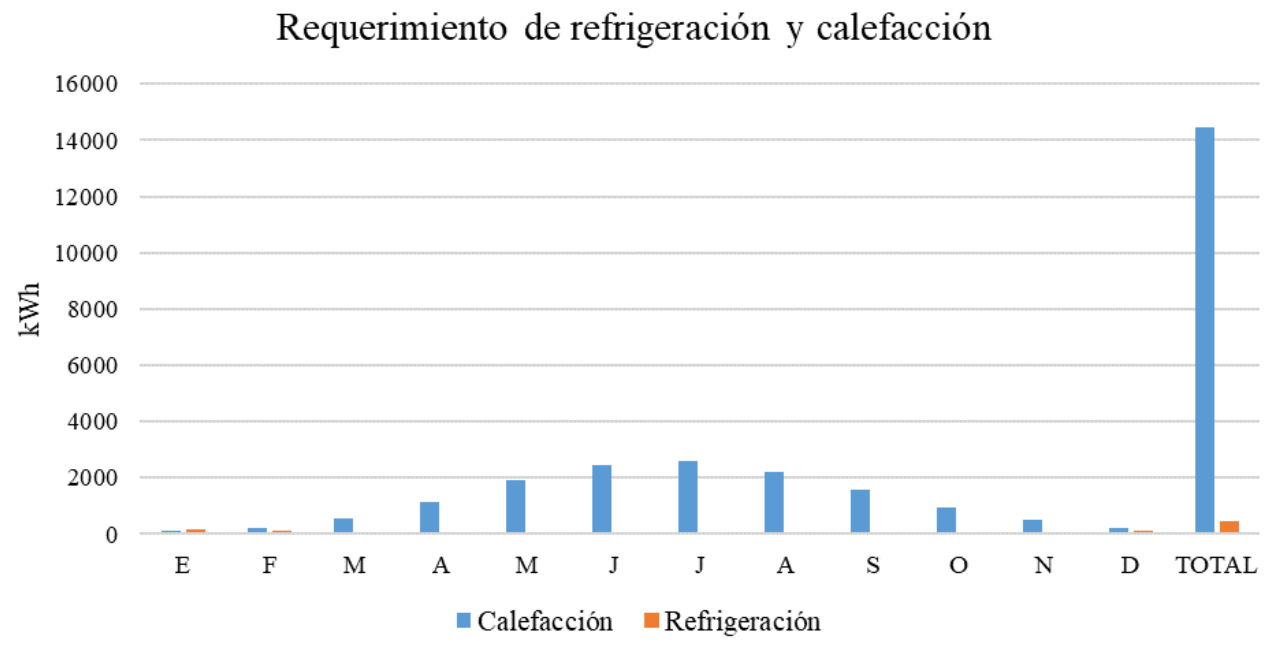

Tabla 10 - Pérdidas y ganancias horarias 1 de enero y 6 de julio en kWh

\begin{tabular}{c|c|c|c|c|c}
\hline & HVAC & $\begin{array}{c}\text { Envolvente } \\
\text { (conducción) }\end{array}$ & $\begin{array}{c}\text { solar } \\
\text { directa }\end{array}$ & $\begin{array}{c}\text { Ventilación e } \\
\text { infiltraciones }\end{array}$ & $\begin{array}{c}\text { Ganancias } \\
\text { internas }\end{array}$ \\
\hline 1 de enero & 38,4 & 7,1 & 5,1 & 12,7 & 7,7 \\
\hline 6 de julio & $-129,4$ & $-52,1$ & 4,9 & $-138,0$ & 7,7 \\
\hline
\end{tabular}

En verano para el día de mayor consumo energético ( $1^{\circ}$ de enero) se observa el mayor aporte de energía de $38,4 \mathrm{kWh}$ por parte del HVAC, seguido por los $12,7 \mathrm{kWh}$ por ventilación e infiltraciones. En invierno para el 6 de julio (día más frío y de mayor consumo) se observa que las mayores pérdidas de energía están determinadas por las ventilaciones e infiltraciones. El aporte de energía por parte del sistema HVAC es de $129,4 \mathrm{kWh}$ lo que sugiere un comportamiento menos favorable en invierno que en verano.

\section{Comparación de los resultados de la simulación térmica con ECOTECT y SIMEDIF para Windows}

El hecho de contar con los resultados de dos simulaciones permite verificar el desempeño de ambos modelos tomando como referencia los datos reales del consumo de energía de la vivienda. Dado que SIMEDIF permite realizar simulación diaria, se toman los datos de temperatura y de radiación para el día más frío promedio para invierno (6 de julio) y el día más caluroso promedio para verano (31 de enero) obtenidos previamente en ECOTECT desde el subprograma Weather Tool $^{12}$. Se introducen los

${ }^{12}$ Weather Tool 2011-CAutodesk, Inc.2010, en base a los datos de la Estación Meteorológica Santa Rosa Aero. mismos datos de los componentes tecnológicos con sus coeficientes de conductividad térmica, ganancias internas en $\mathrm{W}$ y las renovaciones de aire. Se tiene en cuenta la superficie de radiación que incide en cada pared según el análisis de asoleamiento sobre el modelo en tres dimensiones elaborado en ECOTECT para ambos períodos. Para obtener las temperaturas internas "reales" de los locales en ECOTECT y poder hacer una comparación pertinente con SIMEDIF se elimina el sistema de acondicionamiento de "modo mixto", es decir, la vivienda no está acondicionada por medios mecánicos ni pasivos (de no eliminarse el sistema, las temperaturas que arrojaría el software se mantendrían en un rango de entre $24,5^{\circ} \mathrm{C}$ a $27,5^{\circ} \mathrm{C}$, fijado anteriormente como banda de confort).

$\mathrm{Al}$ analizar las temperaturas internas de los locales obtenidas con SIMEDIF se observa que en verano se alcanzan temperaturas máximas de $31,0{ }^{\circ} \mathrm{C}$ y mínimas de $25,0^{\circ} \mathrm{C}$ (Figura 6 superior izquierda). Las curvas en invierno oscilan a la par de la temperatura exterior, pero muy por debajo de la zona de confort, alcanzando temperaturas de 1,5 ${ }^{\circ} \mathrm{C}$ y $5,0^{\circ} \mathrm{C}$, mínimas y máximas respectivamente (Figuras 6 inferior).

Comparando los gráficos obtenidos con ambos programas de simulación,se puede observar un desempeño térmico similar: en la Figura 6 superior (A1), con SIMEDIF las temperaturas de

226 Mazzocco, M. P.; Filippín, C.; Sulaiman, H.; Larsen, S. F. 
todos los locales tienen un comportamiento homogéneo, con una temperatura media interior de $27,6{ }^{\circ} \mathrm{C}$ muy cercana a la de $27,3{ }^{\circ} \mathrm{C}$ de ECOTECT (Figura 6, B1) Para el 6 de julio (Figura 6, A2 y B2) ambos modelos presentan similares temperaturas internas, a excepción de la cocina en ECOTECT, que presenta una diferencia $2{ }^{\circ} \mathrm{C}$ más con respecto a la media. Con un termostato fijado en $24,5^{\circ} \mathrm{C}$ para invierno y 27,5 ${ }^{\circ} \mathrm{C}$ para verano, se obtienen las necesidades máximas de energía auxiliar para ambos días. En la Tabla 11 se presentan los resultados y se observa acuerdo en los valores de energía requeridos. De acuerdo a las conversiones pertinentes, el consumo diario de energía a las $8 \mathrm{~h}$ del día 6 de julio es de $1,40 \mathrm{~m}^{3}$. Para un promedio de uso diario de $\mathrm{H}$ alrededor de $8 \mathrm{~h}$ el valor diario alcanzaría $11,2 \mathrm{~m}^{3}\left(0,22 \mathrm{~m}^{3} / \mathrm{m}^{2}\right)$, consumo cercano al promedio del bimestre 4 (Tabla 3 ) y a valores promedios medidos en otros casos de estudio según párrafos anteriores (FILIPPÍN.; SIPOWICZ.; FLORES LARSEN, 2013).

\section{Propuesta de rehabilitación energética de la envolvente según Nivel B y A de la Norma IRAM 11605}

En los primeros párrafos del presente trabajo se fundamentó el uso de los dos modelos de simulación y en acuerdo con los resultados obtenidos por diferentes autores y según las fortalezas descriptas para ECOTECT en particular por Harish y Kumar (2016). La calibración con los consumos reales de energía de los valores de energía requeridos con ambos modelos de simulación permite asumir que la configuración geométrica, la masa térmica, las ganancias solares, las propiedades térmicas de la envolvente, las sombras y las ganancias internas, fueron representadas aceptablemente en el caso de estudio. De este modo, a partir del modelo termo físico obtenido, se considera la rehabilitación energética de la vivienda partiendo de dos estrategias: solarización y conservación de la energía través del aumento del área efectiva de vidrio en las ventanas ubicadas en el muro norte y del mejoramiento de la resistencia térmica del techo, de la pared ESTE y el cambio de carpintería (marco de aluminio y doble vidrio hermético DVH).

Figura 6 - Superior izquierda (A1): $1^{\circ}$ de enero SIMEDIF (A sup), sup. Derecha (B1): ECOTECT (B sup). Inferior izquierda (A2): 6 de julio SIMEDIF (A inf), inferior derecha (B2): ECOTECT (B inf)

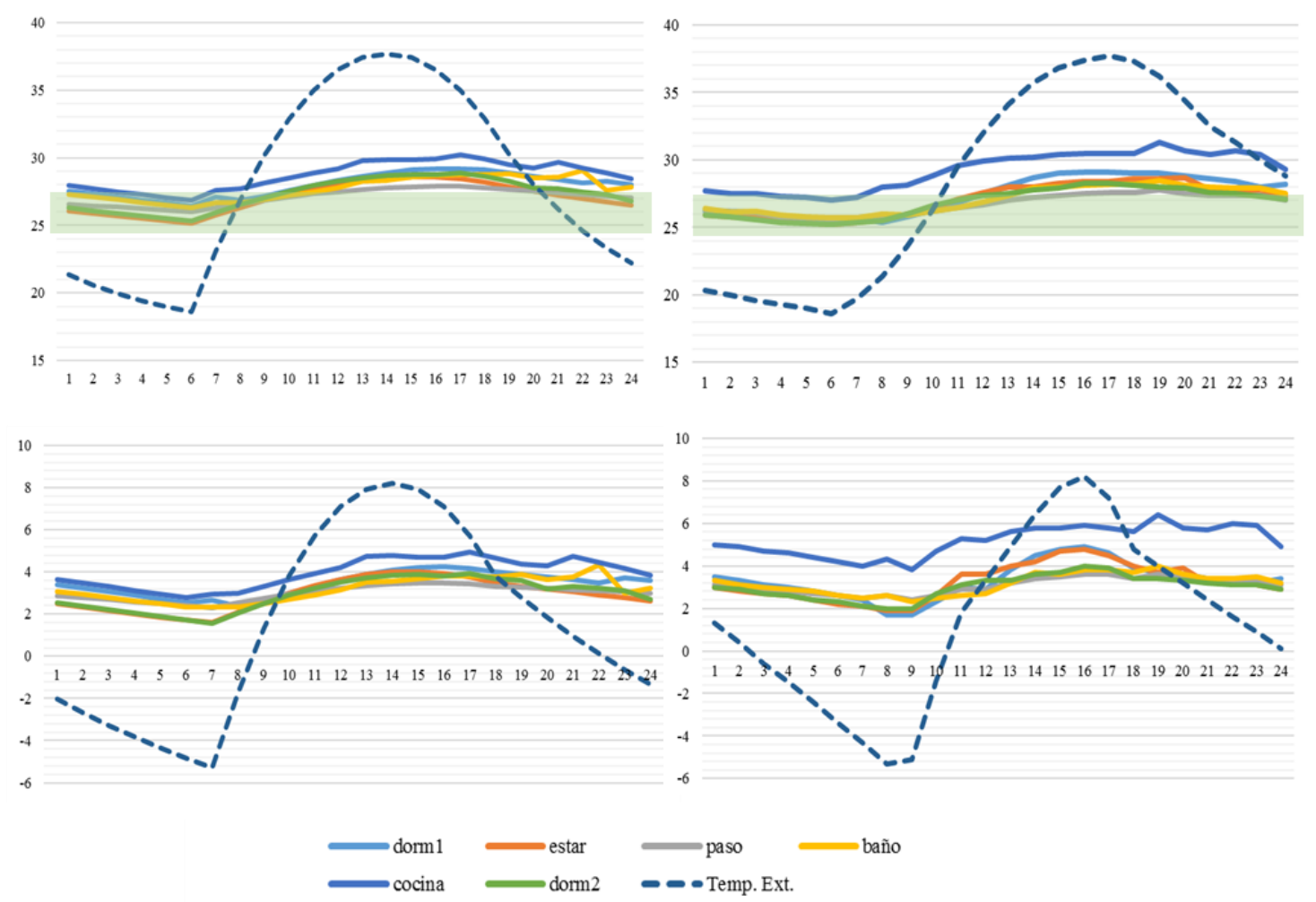

Nota: los gráficos muestran la temperatura de los locales de la vivienda: dormitorio 1, dormitorio 2, paso, baño, cocina y estar y la temperatura exterior. 
Tabla 11 - Comparación de valores obtenidos con los programas SIMEDIF - ECOTECT

\begin{tabular}{l|l|r|r}
\hline & & SIMEDIF & ECOTECT \\
\hline \multirow{3}{*}{6 de julio (8h) } & $\mathrm{kWh}$ & 13,9 & 13,0 \\
& $\mathrm{kcal}$ & 11919,6 & 11180,0 \\
& $\mathrm{~m} \mathrm{~m}^{3}$ (gas natural) & 1,4 & 1,3 \\
\hline \multirow{2}{*}{$1^{\mathrm{o}}$ de enero (15h) } & $\mathrm{kWh}$ & $-4,1$ & $-4,0$ \\
& Frig. & $-3543,2$ & $-3465,8$ \\
\hline
\end{tabular}

La rehabilitación se plantea en primer lugar para satisfacer el nivel B, en los muros y en los techos de la Norma IRAM y en segundo lugar para alcanzar nivel A, y para una nueva banda de confort. La verificación de la Norma IRAM para la envolvente horizontal y vertical, para verano e invierno se muestra en la Tabla 12 .

\section{Nivel B y C de la Norma IRAM 11605}

Intervención en los componentes tecnológicos:

(a) envolvente ESTE: adicionarle una capa de poliestireno expandido $\left(\right.$ densidad $\left.=20 \mathrm{~kg} / \mathrm{m}^{3}\right) \mathrm{de}$ $5 \mathrm{~cm}$ de espesor sobre la cara externa del muro, con una terminación de base coat. El nuevo muro posee una transmitancia térmica $(\mathrm{K})$ de $0,55 \mathrm{~W} / \mathrm{m}^{2} \mathrm{~K}$ que verifica en los niveles $\mathrm{B}$ y $\mathrm{C}$ de la norma IRAM 11605 (INSTITUTO..., 1996) (Tabla 10). El retardo y el y amortiguamiento es de $8 \mathrm{~h}$ y 0,08 respectivamente;

(b) techo: se propone colocar $10 \mathrm{~cm}$ de poliestireno expandido $\left(\right.$ densidad $\left.=20 \mathrm{~kg} / \mathrm{m}^{3}\right)$ y carpeta de nivelación sobre la cubierta tradicional existente. La transmitancia térmica disminuye a $0,30 \mathrm{~W} / \mathrm{m}^{2} \mathrm{~K}$, verificando en los niveles B y C de la norma tanto para invierno como para verano con un retardo y amortiguamiento de $15 \mathrm{~h}$ y 0,08 respectivamente; $\mathrm{y}$

(c) ventanas: cambiar las existentes de madera por aberturas con carpintería de aluminio con ruptor de puente térmico y doble vidriado hermético (DVH 6+126+4), lo que permite disminuir el $\mathrm{K}$ a $2,70 \mathrm{~W} / \mathrm{m}^{2} \mathrm{~K}$. Además, se opta por aumentar el tamaño de las mismas en la fachada NORTE pasando de $1,20 \mathrm{~m}^{2}$ a $2,25 \mathrm{~m}^{2}$. El área de ganancia directa al norte absorbe un 33,6\% de la pared y corresponde a un $16,8 \%$ del área pasiva y al $9 \%$ del área útil de la vivienda. Al efectuar este cambio se establece para la simulación un valor de $0,50 \mathrm{~m}^{3} / \mathrm{h}$ en renovaciones, estimada según Norma IRAM 11604 (INSTITUTO..., 2011) para una carpintería de elevada hermeticidad de acuerdo a la Norma IRAM 11507-1 (INSTITUTO..., 2001).
De acuerdo a las características geométricas y los indicadores energéticos de la intervención de la envolvente, se puede disminuir el Coeficiente global de pérdidas $\mathrm{G}$ original de 3,06 a $1,46 \mathrm{~W} / \mathrm{m}^{3 \circ} \mathrm{C}$, valor que permite satisfacer los requerimientos de la Norma IRAM 11604 (INSTITUTO..., 2011) $\left(\mathrm{G}_{\mathrm{adm}}=1,88 \mathrm{~W} / \mathrm{m}^{3} \mathrm{~K}\right) . \quad \mathrm{Se}$ realiza nuevamente la simulación con los elementos constructivos propuestos y se obtienen las necesidades anuales de AC y H (Tabla 13). Se observa una disminución de necesidades de $\mathrm{H}$ a $5.617,50 \mathrm{kWh}$ y $336,70 \mathrm{kWh}$ para $\mathrm{AC}$ la vivienda que equivalen a un $61 \%$ y un $26 \%$ menos que la vivienda convencional respectivamente. El requerimiento de $\mathrm{H}$ por metro cuadrado disminuye a $98,80 \mathrm{kWh} / \mathrm{m}^{2}$, un $34 \%$ inferior a los $289,60 \mathrm{kWh} / \mathrm{m}^{2}$ obtenidos en la simulación previa.

Se obtienen las pérdidas y ganancias horarias (Tabla 14) para el día de mayor consumo en verano $\left(1^{\circ}\right.$ de enero) y se observa una disminución de ganancias por HVAC, por conducción y también a través de las ventanas respecto de la CONV (Tabla 10), lo cual demuestra la eficacia de la incorporación de medidas de rehabilitación para ahorro energético. Para el 6 de julio, día de mayor consumo de energía en invierno, los resultados arrojan una disminución en las pérdidas por infiltraciones de alrededor del $80 \%$ respecto a la vivienda CONV (Tabla 10) debido al cambio por aberturas de elevada hermeticidad. Disminuyen también las pérdidas por conducción en un valor de casi el $9 \%$ y aumenta la ganancia solar directa (7\%), como resultado del aumento de superficie vidriada hacia el norte.

La intervención energética significa aumentar la energía contenida en 19605 MJ: 1800,0 - 8076,0 y $9729,0 \mathrm{MJ}\left(392,1 \mathrm{MJ} / \mathrm{m}^{2}\right)$ para el mejoramiento térmico de la envolvente vertical, la cubierta y el cambio de carpintería respectivamente. La intervención significa un $15 \%$ de incremento respecto al valor original $\left(8.779,5 \mathrm{MJ} / \mathrm{m}^{2}\right.$, según Tabla 5). 
Tabla 12 - Verificación de coeficiente K según norma IRAM 11605

\begin{tabular}{|c|c|c|c|c|}
\hline \multirow{2}{*}{\multicolumn{3}{|c|}{ ТЕСНО }} & \multicolumn{2}{|c|}{ Verifica } \\
\hline & & & CONV & RE \\
\hline \multicolumn{3}{|c|}{$\mathrm{K}\left(\mathrm{W} / \mathrm{m}^{2 \mathrm{o}} \mathrm{C}\right)$} & 2,03 & 0,3 \\
\hline \multirow[t]{2}{*}{ Nivel A } & Verano & 0,19 & NO & NO \\
\hline & Invierno & 0,26 & NO & NO \\
\hline \multirow[t]{2}{*}{ Nivel B } & Verano & 0,48 & NO & SÍ \\
\hline & Invierno & 0,67 & NO & Sí \\
\hline \multirow[t]{2}{*}{ Nivel C } & Verano & 0,76 & NO & SÍ \\
\hline & Invierno & 1,00 & NO & Sí \\
\hline \multicolumn{5}{|l|}{ MURO } \\
\hline \multicolumn{3}{|c|}{$K\left(W / m^{2 o} C\right)$} & 2,97 & 0,55 \\
\hline \multirow[t]{2}{*}{ Nivel A } & Verano & 0,5 & NO & NO \\
\hline & Invierno & 0,3 & NO & NO \\
\hline \multirow[t]{2}{*}{ Nivel B } & Verano & 1,25 & NO & SÍ \\
\hline & Invierno & 0,8 & NO & Sí \\
\hline \multirow[t]{2}{*}{ Nivel C } & Verano & 2,0 & NO & SÍ \\
\hline & Invierno & 1,39 & NO & Sí \\
\hline
\end{tabular}

Tabla 13 - Requerimiento anual de H y AC en kWh de la vivienda RE con simulación

\begin{tabular}{c|c|c|c}
\hline & Calefacción & Refrigeración & Total \\
\hline TOTAL & 5617,5 & 336,7 & 5954,3 \\
Por $\mathbf{~ m}^{2}$ & 98,9 & 6,0 & 104,8 \\
\hline
\end{tabular}

Tabla 14 - Pérdidas y ganancias horarias 1 de enero y 6 de julio en kWh

\begin{tabular}{c|r|c|c|c|c}
\hline & HVAC & $\begin{array}{c}\text { Envolvente } \\
\text { (conducción) }\end{array}$ & $\begin{array}{c}\text { Solar } \\
\text { directa }\end{array}$ & $\begin{array}{c}\text { Ventilación e } \\
\text { infiltraciones }\end{array}$ & $\begin{array}{c}\text { Ganancias } \\
\text { internas }\end{array}$ \\
\hline 1 de enero & 16,2 & 5,0 & 3,0 & 2,9 & 7,8 \\
6 de julio & $-50,4$ & $-47,6$ & 5,3 & $-29,0$ & 7,8 \\
\hline
\end{tabular}

\section{Nivel A de la Norma 11605}

Se propone adicionar una capa de poliestireno expandido de 10 y $15 \mathrm{~cm}$ en la envolvente vertical y horizontal, respectivamente $\left(\right.$ densidad $\left.=20 \mathrm{~kg} / \mathrm{m}^{3}\right)$. La intervención energética significa disminuir el valor de $\mathrm{K}$ de la pared y del techo a 0,24 y $0,21 \mathrm{~W} / \mathrm{m}^{2} \mathrm{~K}$, respectivamente. El retardo en la pared aumenta a $10 \mathrm{~h}$ y se mantiene el amortiguamiento. En la envolvente horizontal se mantienen los mismos valores del Nivel B. Siempre a partir del modelo termo físico obtenido, se realiza la simulación, por un lado, con los componentes tecnológicos de la vivienda con Nivel A de la Norma IRAM 11605 (invierno y verano) con dos variantes: sin y con aumento de ganancia solar directa y por otro, con la determinación de la zona de confort a partir de la temperatura de neutralidad indicada por Auliciens (1969). Según Tabla 1 la temperatura de neutralidad es de 20,1 y $25,2^{\circ} \mathrm{C}$ para julio y enero, respectivamente $\quad\left(\mathrm{T}_{\mathrm{n}}=17,8+0,31 *\right.$ Temperatura media). Para una aceptabilidad del $90 \%$ se considera un desvío standard según el autor de + $2,5^{\circ} \mathrm{C}$.
La Tabla 15 muestra los resultados obtenidos con una importante reducción del consumo de energía en H y AC. Por un lado, se observa la influencia de la tecnología (conservación) y de la solarización para la configuración de la vivienda en estudio, por otro lado, también la respuesta a los hábitos de uso del sistema de acondicionamiento de aire. El menor consumo corresponde a la vivienda que alcanza el Nivel A de la Norma IRAM (muro y techo) y con la nueva banda de confort en acuerdo con la temperatura estimada según AULICIES (1969) que acuerdan con valores de temperatura de confort recomendados por las normas IRAM. El ahorro de energía destinada a calefaccionar los espacios sería del $89,4 \%$ entre la vivienda original y la vivienda rehabilitada con estrategias de conservación y solarización y nueva banda de confort.

Aun con el Nivel A de la Norma IRAM y la solarización de acuerdo a la disponibilidad de la fachada norte y el área útil de los locales, y una reducción considerable del coeficiente global de pérdidas ' $\mathrm{G}$ ' $(62,3 \%)$ con un nuevo valor $\left(1,13 \mathrm{~W} / \mathrm{m}^{3 o} \mathrm{C}\right)$ que es inferior al admisible según 
Norma IRAM 11601 (INSTITUTO..., 2002) $\left(1,88 \mathrm{~W} / \mathrm{m}^{3 \circ} \mathrm{C}\right)$, no es posible alcanzar el nivel Passivhaus para calefacción ${ }^{13}$. El aumento del área de ventana al norte (opción 3.1 de RE), genera un aumento en la carga pico a las $6 \mathrm{~h}$ en el mes de julio de 2,9\%, pudiendo acordar con Persson, Roos y Wall (2006) quienes concluyen en el trabajo sobre la influencia del área de la ventana en el balance de energía en función de los resultados obtenidos en la simulación de viviendas en Suecia. El valor obtenido en el presente trabajo se eleva al $34,5 \%$ para el mes de enero a las $13 \mathrm{~h}$. Si se considera la opción 3.3 de RE (modificación banda de confort y disminución valor de G) los resultados muestran una importante reducción de la carga pico en $\mathrm{H}(23,6 \%)$ con un aumento de la carga pico en AC a las 13h en enero (37\%). Según la Memoria y Balance de la cooperativa popular de electricidad, Santa Rosa, Ltda. (COOPERATIVA..., 2016) el 49\% de la energía consumida corresponde al sector residencial y la energía facturada tuvo un crecimiento de $4,7 \%$ respecto al balance anterior. Según un artículo en el diario local y exactamente a las 13 horas de diciembre 2013 la demanda de energía del sistema interconectado de Santa Rosa alcanzó un récord histórico (DIARIO TEXTUAL, 2018). Ambas informaciones son válidas para resaltar los resultados del presente trabajo, más aún, el horario de la carga pico en verano que surge de la simulación, coincide con el artículo de divulgación, valoraciones que podrían ser considerados en la planificación de la distribución de la energía eléctrica.

\section{Energía contenida y energía operativa resultante en la vivienda rehabilitada y certificación energética de cada propuesta de intervención}

Respecto a la energía contenida, la intervención energética significa aumentar su valor en 19605,0 MJ: 1800,0 para el mejoramiento térmico de la envolvente vertical, 8076,0 para optimización de la cubierta (Nivel B de la Norma IRAM) y 9729,0 MJ y para cambio de carpintería, dando un total de $392,1 \mathrm{MJ} / \mathrm{m}^{2}$. De acuerdo a Tabla 2, el consumo promedio de electricidad absorbe sólo un 4,7\% del total de energía consumida y considerando que el mayor porcentaje en la energía operativa en el caso de estudio corresponde a la $\mathrm{H}(58,2 \%)$ según Filippín et al. (2017), se toma un valor fijo para otros usos de la energía de 31319,0MJ (calentamiento de agua, cocción de alimentos, iluminación, equipamiento). La estimación permite valorar la energía operativa para los distintos niveles de intervención. Como es de esperar, la intervención significaría una reducción importante de la energía operativa. De este modo, el ahorro sería de un 31,2\% entre la vivienda convencional y el primer caso de intervención para un $4,5 \%$ de incremento de la energía contenida $\left(392,10 \mathrm{MJ} / \mathrm{m}^{2}\right)$ respecto al valor original $\left(8799,5 \mathrm{MJ} / \mathrm{m}^{2}\right)$. La reducción de la energía operativa crece al 41,0\% para un aumento del $7 \%$ de la energía contenida $\left(624,2 \mathrm{MJ} / \mathrm{m}^{2}\right)$. La participación de la energía contenida en el total de energía consumida a lo largo de 50 años pasa del 13\% al 15\% (Nivel B) y al $16 \%$ (Nivel A, caso 1 ) y a un $22 \%$ para el Nivel A caso 3 con un ahorro de la energía operativa del $41 \%$.

Tabla 15 - Consumo de energía en la rehabilitación de la vivienda según Figura 1

\begin{tabular}{|c|c|c|c|c|c|c|c|c|}
\hline \multirow{3}{*}{$\begin{array}{c}\text { Opción de } \\
\text { rehabilitación } \\
\text { según Figura } \\
1\end{array}$} & \multicolumn{6}{|c|}{ Consumo de energíasegúnsimulación (kWh) } & \multicolumn{2}{|c|}{$\begin{array}{c}\text { Consumo } \\
\text { pico } \\
(\mathbf{k W h})\end{array}$} \\
\hline & \multirow{2}{*}{$\begin{array}{c}\text { Calefacción } \\
\text { (C) }\end{array}$} & \multirow{2}{*}{$\begin{array}{l}\text { Refrigeración } \\
\text { (R) }\end{array}$} & \multirow{2}{*}{$\begin{array}{c}\text { Total } \\
(\mathbf{T})\end{array}$} & \multicolumn{3}{|c|}{$/ \mathbf{m}^{2}$} & \multirow{2}{*}{$\mathbf{C}$} & \multirow{2}{*}{$\mathbf{R}$} \\
\hline & & & & $\mathbf{C}$ & $\mathbf{R}$ & $\mathbf{T}$ & & \\
\hline 3 & 4881,3 & 485,7 & 5367,0 & 85,9 & 8,5 & 94,5 & 5,8 & 1,7 \\
\hline 3.1 & 4193,8 & 547,7 & 4741,6 & 73,8 & 9,6 & 83,5 & 5,9 & 2,3 \\
\hline 3.2 & 3061,6 & 495,2 & 3556,6 & 53,9 & 8,7 & 62,6 & 5,3 & 2,3 \\
\hline 3.3 & 1744,4 & 414,4 & 2158,8 & 30,7 & 7,3 & 38,0 & 4,4 & 2,3 \\
\hline
\end{tabular}

Nota: el consumo pico se da a las 8:00h y 15:00h para $C$ y $R$ respectivamente según resultados que arroja la simulación en ECOTECT.

\footnotetext{
${ }^{13}$ Para que un edificio sea considerado bajo el estándar Passivhaus la demanda de energía de calefacción no debe exceder los $15 \mathrm{kWh} / \mathrm{m}^{2}$ de superficie habitable neta por año.
}

230 Mazzocco, M. P.; Filippín, C.; Sulaiman, H.; Larsen, S. F. 
Los resultados se observan en Tabla 16. El consumo anual por metro cuadrado de superficie útil es de $34,9 \mathrm{kWh} / \mathrm{m}^{2} / \mathrm{y}$. El valor es auspicioso aún no pudiendo alcanzar el Nivel Passivehouse. Para la configuración geométrica de la vivienda ameritaría solarizar el sector sur con el ingreso de energía solar a través de algún dispositivo diseñado para tal fin, previo estudio de su factibilidad tecnológica y económica.

Los resultados confirman las conclusiones de Sartori y Hestnes (2007) quienes argumentan que la energía operativa representa una parte dominante en el consumo total de la energía en 50 años y en una muestra de 60 casas, aún en contextos diferentes. Afirman que la reducción de la energía demandada en la operación de un edificio es el aspecto más importante para un diseño energéticamente eficiente en su ciclo de vida.

De acuerdo a la Norma IRAM 11900, Esquema 1 (INSTITUTO..., 2010) se calcula la etiqueta de la eficiencia energética de la envolvente según los parámetros y valores especificados en la norma. Mediante la etiqueta se califica la eficiencia a través de un sistema comparativo, compuesto por ocho clases de eficiencia energética identificadas por las letras A, B, C, D, E, F, G y H, donde la letra A se adjudica a las envolventes de los edificios más eficientes y la $\mathrm{Ha}$ las menos eficientes. Complementariamente, de acuerdo a las características tecnológicas y térmicas de la envolvente vertical de la vivienda CONV y tomando como referencia la norma IRAM 11900 (INSTITUTO..., 2010), y para una temperatura interior de $22,0^{\circ} \mathrm{C}$, el valor de la variación media ponderada de la temperatura es de alrededor de 4,7 ${ }^{\circ} \mathrm{C}$ valor que supera los $4,0^{\circ} \mathrm{C}$ que sugiere la clase $\mathrm{H}$ (envolvente energéticamente ineficiente). Las medidas de mejora adoptadas permitirían aumentar la eficiencia térmica de la envolvente a clase $\mathrm{D}$ $\left(2,0{ }^{\circ} \mathrm{C}<\tau_{m} \leq 2,5{ }^{\circ} \mathrm{C}\right)$ de la norma siendo $\tau_{\mathrm{m}}$ la variación media ponderada de la temperatura, entre la superficie interior de la envolvente y la temperatura interior de diseño en grados Celsius. Para el Nivel A (INSTITUTO..., 1996) se logra aumentar aún más la eficiencia de la envolvente hasta alcanzar la clase $\mathrm{B}\left(1^{\circ} \mathrm{C}<\tau_{m} \leq 1,5^{\circ} \mathrm{C}\right)$, con una $\tau_{\mathrm{m}}$ de $1,4^{\circ} \mathrm{C}$ sin riesgo de condensación alcanzando temperaturas en la última capa del paramento en el interior de la vivienda que satisface el requerimiento de Passivehouse $\left(17,0{ }^{\circ} \mathrm{C}\right.$ ) (Figura 7).

Tabla 16 - Energía contenida y operativa (50 años de uso de la vivienda CONV y RE)

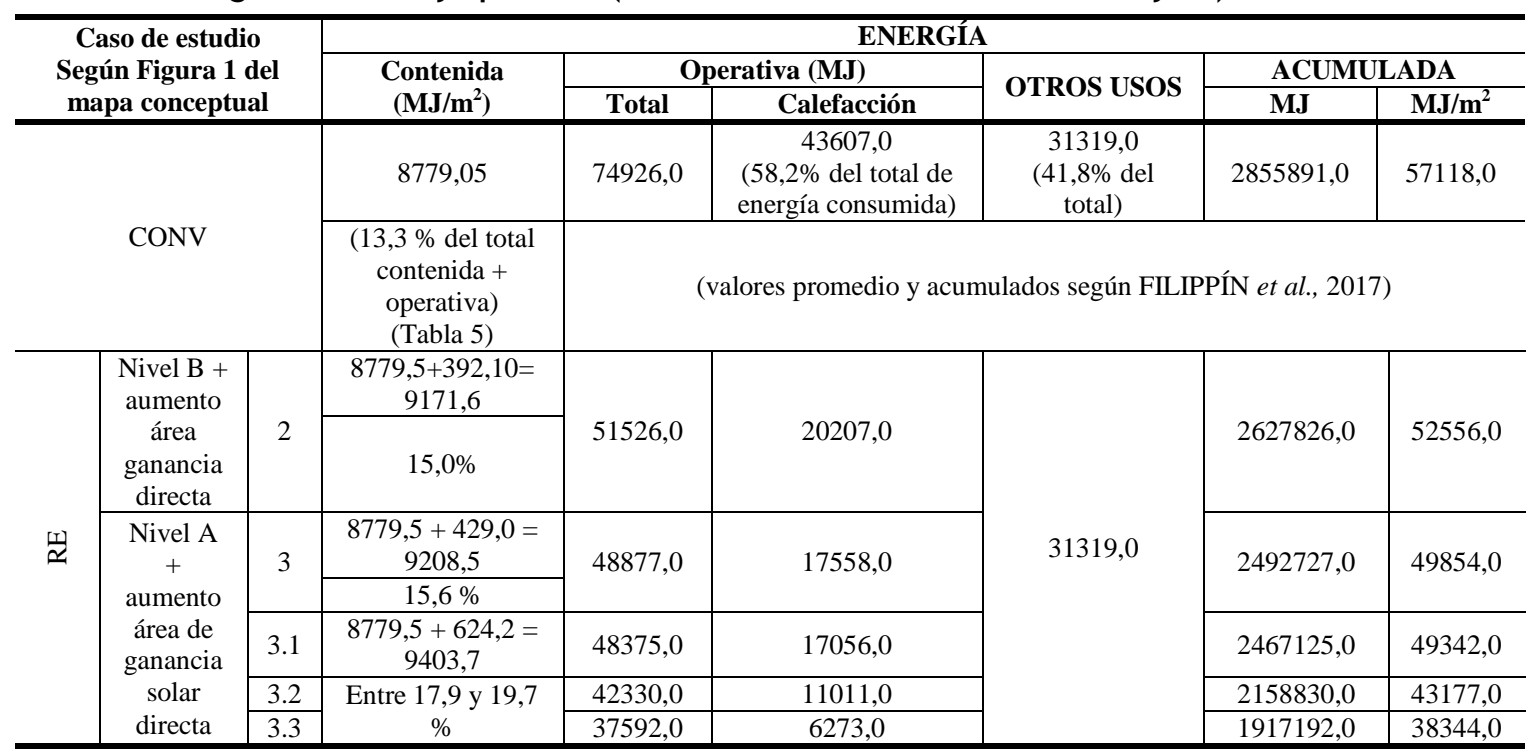


Figura 7 - Comparación etiqueta de eficiencia energética de calefacción
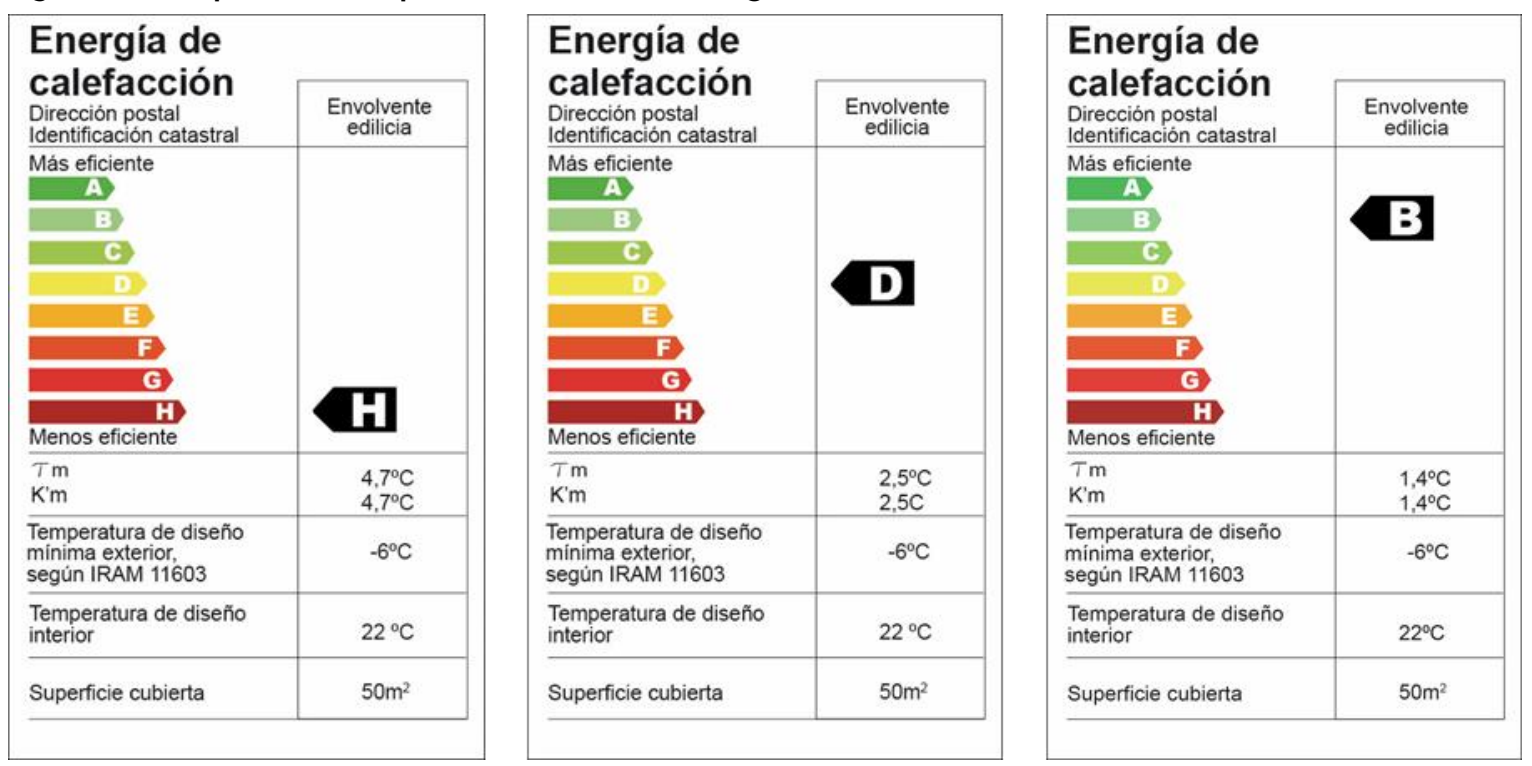

Fuente: elaboración propia en base a norma IRAM 11900 (INSTITUTO..., 2010).

\section{Conclusiones}

El acceso a la documentación técnica y a los consumos de energía durante períodos históricos fue determinante en la elección del caso de estudio, ya que la simulación y cálculos estacionarios se corroboraron (calibraron) según los datos medidos. El desarrollo del trabajo permitió cumplir con el objetivo descripto en el inicio. Se analizó la performance energética, se estimaron indicadores morfológicos, entre ellos FAEP, cuyo valor resultó inferior al definido en la bibliografía como energéticamente eficiente, por lo tanto la envolvente de la vivienda tiene un potencial de intervención adecuado para plantear una rehabilitación energética. Se pudo obtener el modelo termo físico de la vivienda a partir de la simulación y calibración con el consumo real de energía para un período histórico. La obtención del modelo termo físico permitió estudiar el potencial de intervención tecnológica para mejorar energéticamente la vivienda y satisfacer las recomendaciones de las Normas IRAM pertinentes. Se calculó el ahorro de energía y el incremento en la participación relativa de la energía contenida en el total de energía consumida a lo largo de 50 años. Los resultados muestran que la rehabilitación energética de la envolvente de la vivienda en estudio satisface las recomendaciones de las Normas IRAM pertinentes y permite mejorar el nivel del etiquetado energético. A partir de la intervención energética, la energía contenida se incrementa un 4,3 y un $7,1 \%$ para alcanzar el Nivel B y A de la Norma IRAM respectivamente, con un ahorro de energía para ambas intervenciones, del 53,7 y del $85,6 \%$ en la calefacción de los espacios alcanzando ambientes térmicamente aceptables. Los resultados del trabajo ameritan una evaluación económica de acuerdo a los nuevos valores de energía en Argentina y un estudio de la resolución constructiva de los componentes tecnológicos.

\section{Referencias}

ADALBERT,K. Energy Use During the Life Cycle of Single-Unit Dwellings: examples. Building and Environment, v. 32, p. 321-329, 1997.

AULICIENS, A. Effects of Weather on Indoor Thermal Comfort. International Journal Biometeorology, v. 13, 2, p. 147-162, 1969.

AUTODESK. Ecotect. Disponível em: <www.autodesk.com/ecotect-analysis>.Acesso em: 2. jun. 2011.

BEHLING, S.; BEHLING, S. Sol Power, La Evolución de la Arquitectura Sostenible. Barcelona: Gustavo Gili, 2002.

BELMONTE, M. L. et al. Estadísticas Agroclimáticas de la EEA Anguil Ing. Agr. Guillerrmo Covas. Período 1973-2016. Ediciones INTA, 2017.

BOUTET, M. L.; HERNÁNDEZ, A. L.; JACOBO, G. J.Validación de Simulaciones Interactivas Con Simedif y Ecotect, a Partir de Auditorias Energeticas de Un Edificio Escolar de la Ciudad de Resistencia. Avances en Energías Renovables y Medio Ambiente, v. 16, 2012. 
CEPEDA GUTIÉRREZ, M,; MARDARAS

LARRAÑAGA, I. Cuantificación Energética de la Construcción de Edificios y el Proceso de Urbanización. Con Arquitectura, v. 12, p. 65-80, 2013.

CLARKE, J. A. Energy Simulation in Buildings Design. $2^{\text {nd }}$. ed. Oxford: Butterworth-Heinemann, 2001.

COLE, J. Life-Cycle Energy Use in Office Buildings. Buiding and Environment, v. 31, n. 4, p. 307-317, 1996.

CONCRETE CENTRE Y ARUP. DynamicThermal-Properties-Calculator-(ver-1-0). Excel Free Tool based on ISO 13786. 2017.

COOPERATIVA POPULAR DE ELECTRICIDAD. Memoria y Balance General 2014-2015. 83 $^{\circ}$ Ejercicicio Económico y Social. Santa Rosa, 2016.

CRAWFORD, R. Life Cycle Energy Analysis. Environment Design Guide, v. 71 RC, mar. 2012.

CZAJKOWSKI, J.; GOMEZ, A. Introducción al Diseño Bioclimático y Economía Energética Edilicia: fundamentos y métodos. La Plata: Editorial de la Universidad Nacional de La Palta, 1994.

DI BERNARDO, A.; FILIPPÍN, C.; PIPA, D. Monitoreo y Simulación Térmica Energética de Verano de Una Vivienda en Condiciones Reales de Uso en Clima Templado Cálido. Avances en Energías Renovables y Medio Ambiente, v. 15, p. 08.67-08.74, 2011.

\section{DIARIO TEXTUAL Consumo Récord de}

Electricidad en Santa Rosa. Disponible:

$<$ http://www.diariotextual.com/economia/item/117 85-hub-un-consumo-r\%C3\%A9cord-deelectricidad-en-santa-rosa-y-toay.html>. Acceso: 29 jun. 2018.

DIXIT, M. K. et al. Identification of Parameters For Embodied Energy Measurement: a literature review. Energy and buildings, v. 42, n. 8, p. 1238-1247, 2010.

ECOTECT. Ecotect: software de diseño de construcción sustentable. Disponible: $<$ http://www.plataformaarquitectura.cl/cl/0262481/ecotect-software-de-diseno-deconstruccion-sustentable>. Acceso: 29 jun. 2018.

EDWARDS, P. J. et al. Embodied Energy Impact Modelling /or Housing Design. In: ARCOM, 9., Oxford, 1993. Proceedings... Oxford, 1993.
ESTEVES, A.; GELARDI, D. Relación Entre la Ganancia Solar y la Calefacción Auxiliar Para dos Tipos de Envolvente Formal Edilicia. Avances en Energías Renovables y Medio Ambiente, v. 14 p. 33-40, 2010.

ESTEVES, A.; GELARDI, D.; MERCADO, M. V. Evaluación del Costo Monetario y el Costo Energético de Elementos Edilicios Para Edificios Energéticamente Eficientes. In: CONGRESSO INTERNACIONAL DE AR CONDICIONADO, REFRIGERAÇÃO, AQUECIMENTO E VENTILAÇÃO, 9., Porto Alegre, 2014. Anais... Porto Alegre, 2014.

ESTEVES, A.; OLIVA, A. L; GELARDI, D. Nueva Medida Para Considerar la Forma en la Conservación de Energía en Edificios. Revista de la Universidad de Mendoza, v. 16, 1997-1999.

FILIPPÍN, C. et al. Retrospective Analysis of the Energy Consumption of Single-Family Dwellings in Central Argentina: retrofitting and adaptation to the climate change. Renewable Energy, v. 101, p. 1-16, 2017.

FILIPPÍN, C.; FLORES LARSEN, S.; LOPEZ GAY, E. Energy Improvement of a Conventional Housing in Argentina Through Thermal Simulation. Renewable Energy, v. 33, p. 22462257, 2008.

FILIPPÍN,C.; SIPOWICZ, E.; FLORES LARSEN, S.Monitoreo Energético Experimental de Vivienda Unifamiliar Compacta en Un Clima Templado Frío de Argentina. Ambiente Construído, Porto Alegre, v. 13, n. 4, p. 249-267, out./dez, 2013.

FLORES LARSEN S.; FILIPPÍN C.; LESINO G.Thermal Behavior of bullding walls in Summer: Comparison of Available Analytical Methods and Experimental Results For a Case Study. Building Simulation, v. 2, p. 3-18, 2009.

FLORES LARSEN, S. et al.An Experience on Integrating Monitoring and Simulation Tools in the Design of Energy-Saving Buildings. Energy and Buildings, v.40, p. 987-997, 2008.

FLORES LARSEN, S.; LESINO, G. Simedif Bajo Windows. 2001. Available at:

<http://exactas.unsa.edu.ar/simedif/>. Access: 15 jun. 2016.

GONZÁLEZ MORA, R. Vida Útil Ponderada de Edificaciones.Costa Rica, 2005. Tesis Profesional - Ingeniería Universidad de San José, Costa Rica, 2005.

GONZALO, G. Manual de Arquitectura

Bioclimática: programa para el cálculo de transmitancia térmica. CEEMA, IAA, FAU, UNT. Editorial Nobuko. 2003. 
GOULDING, J. R.; LEWIS, J. O.; STEEMERS, T. C. Energy in Architecture: the european passive solar handbook. London: Batsford for the Commission of the European Communities, 1994.

GROSSI GALLEGOS, H.; RIGHINI, R. Atlas de Energía Solar de la República Argentina. Versión CD, 2007.

HARISH, V. S. K. V.; KUMAR, A. A Review on Modeling and Simulation of Building Energy Systems. Renewable and Sustainable Energy Reviews, v. 56, p. 1272-1292, 2016.

INSTITUTO ARGENTINO DE NORMALIZACIÓN Y CERTIFICACIÓN.IRAM 11507-1: carpintería de obra: ventanas exteriores: requisitos básicos y clasificación. Buenos Aires, 2001.

INSTITUTO ARGENTINO DE

NORMALIZACIÓN Y CERTIFICACIÓN.

IRAM 11601: aislamiento térmico de edificios, métodos de cálculo:propiedades térmicas de los componentes y elementos de construcción en régimen estacionario. Buenos Aires, 2002.

INSTITUTO ARGENTINO DE NORMALIZACIÓN Y CERTIFICACIÓN. IRAM 11603: clasificación bioambiental de la República Argentina. Buenos Aires, 1996a.

INSTITUTO ARGENTINO DE NORMALIZACIÓN Y CERTIFICACIÓN. IRAM 11604: aislamiento térmico de edificios: verificación de sus condiciones higrotérmicas: ahorro de energía en calefacción: coeficinte volumétrico $\mathrm{G}$ de pérdidas de calor: cálculo y valores límites. Buenos Aires, 2011.

INSTITUTO ARGENTINO DE NORMALIZACIÓN Y CERTIFICACIÓN. IRAM 11605: acondicionamiento térmico de edificios, condiciones de habitabilidad en edificios: valores máximos de transmitancia térmica en cerramientos opacos. Buenos Aires, 1996b.

INSTITUTO ARGENTINO DE NORMALIZACIÓN Y CERTIFICACIÓN. IRAM 11900: etiqueta de eficiencia energética de calefacción para edificios: clasificación según la transmitancia térmica de la envolvente. Buenos Aires, 2010.

INTERNATIONAL ENERGY AGENCY. Transition to Sustainable Buildings, Strategies and Opportunities to 2050. IEA, 2013.

INTERNATIONAL ORGANIZATION DE STANDARDIZATION. ISO 7730: ergonomics of the thermal environment: analytical determination and interpretation of thermal comfort using calculation of the PMV and PPD indices and local thermal comfort criteria. Pennsylvania, 2005.
MA, Z. et al. Existing Building Rertrofits: methodoloy and state-of-the-art. Eenergy and Buildings, v. 55, p. 889-902, 2012.

MASCARÓ, J. L. Variación de los Costos de los Edificios Con las Decisiones Arquitectónicas. La Plata: UNLP, FAU, 1983.

NEJAT, P. et al. A Global Review of Energy Consumption, $\mathrm{CO} 2$ Emissions and Policy in the Residential Sector (with an overview of the top ten $\mathrm{CO} 2$ emitting countries). Renewable and Sustainable Energy Reviews, v. 43, p. 843-862, 2015.

NIELSEN P. et al. Energi- og miljöanalyser af bygninger (In Danish). Energy and environmental analysis of buildings. SBImedelelse 108. Hörsholm: Danish Building Research Institute, 1995.

PERSSON, M.L.; ROOS, A.; WALL, M. Influence of Windows Size on the Energy Balance of Low Energy Houses.Energy and Buildings, v 38, P. 181-188, 2006.

SANTAMOURIS, M. et al. Passive and Active Cooling For the Outdoor Built Environment: analysis and assessment of the cooling potential of mitigation technologies using performance data from 220 large scale projects. Solar Energy. Available: <https://doi.org/10.1016/j.solener.2016.12.006>. Access: 19 dec. 2006.

SARTORI, I.; HESTNES, A. G. Energy Use in the Life Cycle of Conventional and Low-Energy Buildings: a review article. Energy and Buildings, v. 39, p. 249-257, 2007.

\section{SECRETARÍA DE ENERGÍA; MINISTERIO DE} PLANIFICACIÓN. Balance Energético

Nacional. [Reporte]. Tecnología de la Información - Secretaría de Energía. Argentina, 2012.

THORMARK, C.A Low Energy Building in a Life Cycle: its emided energy, energy need for operation and recycling potential. Building and Environment, v. 37, p. 429-435, 2002.

VERGARA, G.; CASAGRANDE, G. Estadísticas Agroclimáticas de la Facultad de Agronomía, Santa Rosa, La Pampa, Argentina 1977/2010. v.22 Suplemento $N^{\circ} 1$, Revista de la Facultad de Agronomía UNLPam, 2012.

WINTER, B. N.; HESTNES, A. G. Solar versus Green: the analysis of a Norwegian row house. Solar Energy, v. 66, n. 6, p. 387-393, 1999.

YAÑEZ PARAREDA, G. Energía Solar, Edificación y Clima: tomo I. Ministerio de Obras Públicas y Urbanismo, España, 1982. 
ZABALZA BRIBIAN, I. et al. Life Cycle Assessment in Buildings: state-of-the-art and simplified LCA methodology as a complement for building certification. Building and

Environment,v. 44, n. 12, p. 2510-2520, 2009.

ZUO, J.; ZHAO, Z. Y. Green Building ResearchCurrent Status and Future Agenda: a review. Iew, v. 30, p.271-281, 2014

\section{Agradecimentos}

Este trabajo fue parcialmente financiado por el Ministerio de Ciencia, Tecnología eInnovación Productiva de la Nación (MINCYT-ANPCYT PICT 2014-2605). Nuestro agradecimiento al Dr. Alvaro Di Bernardo por sus aportes.

\section{Maria Pia Mazzocco}

Arquitecta | Lima, 1566 | Córdoba - Argentina | CP 500 | Tel.: +54-(9351)-650-9685 | E-mail: piamazzocco@gmail.com

\section{Celina Filippín}

Consejo Nacional de Investigaciones Científicas y Técnicas | Av. Spinetto, 785 | 6300 | Santa Rosa - La Pampa - Argentina | Tel.: +54(952)-434-222 | E-mail: cfilippin@cpenet.com.ar

\section{Halimi Sulaiman}

Consejo Nacional de Investigaciones Científicas y Técnicas | Centro Experimental de Vivienda Económica | Igualdad, 3535 | Córdoba Argentina | CP 5000 | Tel.: +54 (9)-264-5461041 | E-mail: halimi.sulaiman@gmail.com

\section{Silvana Flores Larsen}

Instituto de Investigaciones em Energía No Convencional | Universidad Nacional de Salta | Av. Bolivia, 5150 | 4400 | Salta - Argentina | E-mail: seflores@unsa.edu.ar

\section{Revista Ambiente Construído}

Associação Nacional de Tecnologia do Ambiente Construído

Av. Osvaldo Aranha, $99-3^{\circ}$ andar, Centro

Porto Alegre - RS - Brasil

$$
\text { CEP } 90035-190
$$

Telefone: +55 (51) 3308-4084

Fax: +55 (51) 3308-4054

www.seer.ufrgs.br/ambienteconstruido

E-mail: ambienteconstruido@ufrgs.br

This is an open-access article distributed under the terms of the Creative Commons Attribution License. 